\title{
Convective heat transfer enhancement in low Reynolds number flows with wavy walls
}

\author{
Fernando V. Castellões a , João N.N. Quaresma ${ }^{\text {b,c }}$, Renato M. Cotta ${ }^{\text {,** }}$ \\ ${ }^{a}$ Petrobras RED Center - CENPES, Rio de Janeiro, RJ, Brazil \\ ${ }^{\mathrm{b}}$ School of Chemical Engineering, Universidade Federal do Pará - FEQ/UFPA, Belém, PA, Brazil

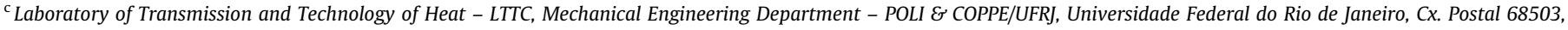 \\ Rio de Janeiro, RJ 21945-970, Brazil
}

\section{A R T I C L E I N F O}

\section{Article history:}

Received 14 April 2009

Received in revised form 14 December 2009

Accepted 14 December 2009

Available online 3 February 2010

\section{Keywords:}

Low Reynolds number flows

Micro-channels

Wavy walls

Heat transfer enhancement

Forced convection

Integral transforms

\begin{abstract}
A B S T R A C T
The present work reports the analysis of combining low Reynolds number flows and channels with wall corrugation and the corresponding thermal exchange intensification achieved. The proposed model involves axial heat diffusion along the fluid and adiabatic regions both upstream and downstream to the corrugated heat transfer section, in light of the lower values of Reynolds numbers (and consequently Peclet numbers) that can be encountered in the present class of problems. Aimed at developing a fast and reliable methodology for optimization purposes, the related laminar velocity field is obtained by an approximate analytical solution valid for smooth corrugations and low Reynolds numbers, typical for instance of micro-channel configurations, locally satisfying the continuity equation. A hybrid numerical-analytical solution methodology for the energy equation is proposed, based on the Generalized Integral Transform Technique (GITT) in partial transformation mode for a transient formulation. The hybrid approach is first demonstrated for the case of a smooth parallel-plates channel situation, and the importance of axial heat conduction along the fluid is then illustrated. Heat transfer enhancement is analyzed in terms of the local Nusselt number and dimensionless bulk temperature along the heat transfer section. An illustrative sinusoidal corrugation shape is adopted and the influence of Reynolds number and corrugation geometric parameters is then discussed.
\end{abstract}

(C) 2010 Elsevier Ltd. All rights reserved.

\section{Introduction}

Within the scope of thermal engineering, energy conservation and sustainable development demands have been driving research efforts towards more energy efficient equipments and processes. The petroleum and process industries have been quite active in progressively incorporating heat transfer enhancement solutions to the efficiency increase requirements along the years [1]. More recently, heat exchangers employing micro-channels with characteristic dimensions below 500 microns have been calling the attention of researchers and practitioners, towards applications that require high heat removal demands and/or space and weight limitations [2]. Motivated by the search for optimal solutions in heat exchange rates, Steinke and Kandlikar [3] critically analyzed various heat transfer enhancement techniques as applied to the micro-channels scale. Among the passive enhancement techniques then discussed, the authors emphasize the utilization of treated surfaces, rough or corrugated walls and additives for working fluids. Several other approaches were disregarded in light of the dif-

\footnotetext{
* Corresponding author. +55 215562 5566; fax: +55 2155628383 .

E-mail address: cotta@mecanica.ufrj.br (R.M. Cotta).
}

ficulties in manufacturing or mechanically modifying the thermal system at the micro-scale. In parallel, a few previous works have addressed the interest in investigating channel corrugations at the micro-scale, either for liquid or gaseous flows [4,5].

The research here reported addresses the convective heat transfer in low Reynolds number flows within channels possibly enhanced by the presence of axial heat conduction along the fluid and wall corrugations. First, the typical low Reynolds numbers in applications such as micro-systems may lead to low values of the Peclet number that bring up some relevance to the axial heat diffusion along the fluid stream, especially for regions close to the inlet. Then, both the upstream and downstream sections of the micro-channel that are not actually part of the heat transfer section, may participate in the overall heat transfer process, and finally yield different predictions than those reached by making use of conventional macro-scale relations for ordinary liquids or gases. Therefore, our first objective is to inspect such effects of the axial heat diffusion within the fluid. For this reason, it was initially necessary to identify the range of governing parameters to be analyzed, in order to allow for an appropriate modeling of the relevant physical phenomena that may appear at this dimensional scale. Second, either due to the inherent difficulties in achieving 


\section{Nomenclature}

$A_{i j}, B_{n i}, C_{m n}$ coefficients defined by Eqs. (24a), (24b) and (24c), respectively

$d \quad$ half the distance between the flat walls

$D_{m n}, E_{m n}$ coefficients defined by Eqs. (24d) and (24e), respectively

$f y_{0}^{*}, f y_{0} \quad$ function that describes lower wall boundary, dimensional and dimensionless, respectively

$f y_{1}^{*}, f y_{1}$ function that describes upper wall boundary, dimensional and dimensionless, respectively

$F_{m n} \quad$ coefficient defined by Eq. (24f)

$g \quad$ source function defined by Eq. (11g)

$\bar{g}_{m} \quad$ coefficient defined by Eq. (24h)

$G_{m n} \quad$ coefficient defined by Eq. (24g)

$L^{*}, L \quad$ total length of the channel, dimensional and dimensionless, respectively

$L_{1}^{*}, L_{1} \quad$ length of region 1 , dimensional and dimensionless, respectively

$L_{2}^{*}, L_{2} \quad$ total length of the regions 1 and 2, dimensional and dimensionless, respectively

MS sub-domain discretization parameter (maximum allowed step size)

$\mathbf{n}_{y_{0}}, \mathbf{n}_{y_{1}} \quad$ unit normal vector associated with the lower and upper wall boundaries, respectively

$N_{1}, N_{2}, N_{3}$ truncation orders for the temperature expansions in regions 1,2 and 3, respectively

$N u_{0}, N u_{1}$ local Nusselt numbers associated with the lower and upper wall boundaries, respectively

$N \psi_{i}, N \Gamma_{m}$ norms defined by Eqs. (16e) and (17e), respectively

$\mathrm{Pe} \quad$ Peclet number

Re Reynolds number

$t^{*}, t \quad$ time variable, dimensional and dimensionless, respectively

$T^{*}, T_{A}$ temperature field, dimensional and dimensionless, respectively

$T_{m} \quad$ bulk temperature
$\bar{T}_{1, i}, \bar{T}_{2, m}, \bar{T}_{3, i}$ transformed potentials for the regions 1,2 , and 3 , respectively

$T_{w, 0}^{*}, T_{w, 1}^{*}$ temperatures of the lower and upper wall boundaries, respectively

$T_{A, w, 1}$ dimensionless temperature of the upper wall boundary

$u^{*}, u$ longitudinal velocity component, dimensional and dimensionless, respectively

$u_{m} \quad$ average velocity

$v^{*}, v$ transversal velocity component, dimensional and dimensionless, respectively

$x^{*} \quad$ longitudinal coordinate

$x_{1}, x_{2}, x_{3}$ normalized longitudinal coordinates

$y^{*}, y \quad$ transversal coordinate, dimensional and dimensionless, respectively

\section{Greek letters}

$\alpha^{*}, \alpha \quad$ channel amplitude, dimensional and dimensionless, respectively

$\alpha_{f} \quad$ fluid thermal diffusivity

$\beta_{m} \quad$ eigenvalues defined by Eq. (17f)

$\Gamma_{m} \quad$ eigenfunctions defined by Eq. (17d)

$\Delta T_{c} \quad$ reference temperature difference

$\mu_{i} \quad$ eigenvalues defined by Eq. (16f)

$\xi \quad$ dimensionless longitudinal coordinate

$\phi \quad$ filtered potential

$\bar{\phi}_{m} \quad$ coefficient defined by Eq. (24i)

$\psi_{i} \quad$ eigenfunctions defined by Eq. (16d)

Subscripts and superscripts

- integral transformed quantities

$\bar{i}, j, m, n$ expansions indices

$1,2,3$ relative to the regions 1,2 , and 3 , respectively smooth surfaces during micro-fabrication processes or to the actual purpose of improving mixing and/or heat transfer, microchannels with irregularly shaped walls started gaining some focus in the heat and mass transfer literature, as pointed out in the brief review above. Thus, the analysis of laminar forced convection within micro-channels with corrugated walls, and the possible heat transfer enhancement effect achieved, is another goal of the present study.

The steady two-dimensional flow problem was handled by adopting an approximate analytical solution that essentially adapts the fully developed velocity profile to the wall geometric variations, satisfying the fully developed momentum equation and the continuity equation [6]. Such simplified approach was introduced in [6] aimed at the solution of incompressible flow problems in low Reynolds number situations and gradual geometric variations in converging or diverging walls. The solution methodology for the energy equation first introduces a domain decomposition strategy, redefining the coordinates systems for the three heat transfer regions, so as to rewrite the problem in the form of a system of equations within the same mathematical domain, coupled at the interfaces of the three regions. Then, a hybrid numerical-analytical solution based on the Generalized Integral Transform Technique (GITT) is proposed [7-9], which consists on the elimination of the transversal coordinate by integral transformation, and results in a coupled system of onedimensional partial differential equations for the transformed temperatures. This system is then handled numerically with local error control by making use of the Method of Lines implemented in the Mathematica system [10]. This so-called partial transformation mode of the GITT allows for the accurate and flexible solution of multidimensional partial differential systems [11] and has been previously employed in the solution of transient and periodic forced convection within smooth parallel-plates micro-channels [12-14]. This alternative hybrid solution strategy to the more usual full integral transformation mode is of particular interest in the treatment of transient convection-diffusion problems with a preferential convective direction. In such cases, the partial integral transformation in all but one space coordinate, may offer an interesting combination of relative advantages between the eigenfunction expansion approach and the selected numerical method for handling the coupled system of one-dimensional partial differential equations that results from the transformation procedure. It should be recalled that the GITT approach has already been previously applied to the analysis of heat transfer within smooth rectangular micro-channels $[15,16]$ for steady state situations.

The situation of a smooth parallel-plates channel is here first analyzed, for typical values of the governing parameters, so as to provide validations of the hybrid numerical-analytical solution for the energy equation, while also illustrating the importance of the axial heat diffusion along the fluid, especially in the transition from the first adiabatic region and the heat transfer section. Then, the approximate analytical solution of the flow problem is demonstrated, as compared to benchmark results of the two-dimensional Navier-Stokes equations, as obtained from the GITT itself in previ- 
ous works [17]. The illustrative situation of sinusoidal symmetric corrugated walls is considered more closely, allowing for parametric variations on the corrugation geometry. Finally, the heat transfer enhancement is inspected for a few different combinations of flow, thermal and geometric parameters, in terms of both the dimensionless bulk temperature and local Nusselt number along the heat transfer section. The present contribution is thus related to the fundamental analysis of forced convection in low Reynolds number flows, here illustrated by flow within micro-channels as required for the design of micro-heat exchangers, including the effects of axial heat conduction and wall corrugation or roughness on heat transfer enhancement.

\section{Analysis}

We consider transient laminar forced convection within channels formed by smooth or corrugated plates. Three regions along the channel are considered in the problem formulation, as described in Fig. 1 below, which is a general representation of a channel with asymmetric irregular walls. First, an adiabatic region with smooth walls, followed by the heat transfer section with prescribed temperatures at the corrugated walls, and the third one, following the corrugated region, is again made of smooth adiabatic walls.

The two-dimensional steady flow is assumed to be laminar and incompressible, with temperature independent thermophysical properties, while viscous dissipation and natural convection effects are neglected. Also, the continuum hypothesis is considered to be valid throughout the flow region, without slip or temperature jump at the boundaries, such as in the case of liquids flowing in microchannels. Due to the possible low values of Peclet number, in light of the lower range of Reynolds numbers, axial diffusion along the fluid is not disregarded. Also, the flow is assumed to be fully developed at the first section entrance, but varies along the axial coordinate once the corrugated section is reached.

In obtaining the velocity field along the flow, the full NavierStokes equations should be employed, yielding the variable velocity components and pressure field along the transversal and longitudinal directions, as recently demonstrated in [17], where the Generalized Integral Transform Technique (GITT) has been employed in the hybrid numerical-analytical solution of this laminar flow. However, for sufficiently low Reynolds numbers and smooth variations on the wall corrugations, an approximate solution has been previously proposed [6], essentially by accounting for the variable cross section within the local mass balance equation in a qua- si-fully developed formulation, thus neglecting the inertia terms influence on the velocity components modification. These explicit solutions for the velocity components are particularly handy, especially in pre-design and optimization tasks, and shall be here exploited to demonstrate the transient thermal problem solution. Thus, following the approach in [6], and for sufficiently smooth wall geometries defined by the $x^{*}$ functions $f y_{0}^{*}$ and $f y_{1}^{*}$, as depicted in Fig. 1 , the velocity components are analytically derived as:

$$
\begin{aligned}
u^{*}\left(x^{*}, y^{*}\right)= & \frac{-\left(y_{0}^{*}-y_{1}^{*}\right)}{\left[f y_{0}^{*}\left(x^{*}\right)-f y_{1}^{*}\left(x^{*}\right)\right]^{3}}\left\{-y^{* 2}+y^{*}\left[f y_{0}^{*}\left(x^{*}\right)+f y_{1}^{*}\left(x^{*}\right)\right]\right. \\
& \left.-f y_{0}^{*}\left(x^{*}\right) f y_{1}^{*}\left(x^{*}\right)\right\} \\
v^{*}\left(x^{*}, y^{*}\right)= & \frac{-\left(y_{0}^{*}-y_{1}^{*}\right)}{\left[f y_{0}^{*}\left(x^{*}\right)-f y_{1}^{*}\left(x^{*}\right)\right]^{4}}\left\{6\left[y-f y_{0}^{*}\left(x^{*}\right)\right]\left[y-f y_{1}^{*}\left(x^{*}\right)\right]\right. \\
& \left.\times\left\{f y_{1}^{* \prime}\left(x^{*}\right)\left[y-f y_{0}^{*}\left(x^{*}\right)\right]-f y_{0}^{* \prime}\left(x^{*}\right)\left[y-f y_{1}^{*}\left(x^{*}\right)\right]\right\}\right\}
\end{aligned}
$$

It can be noticed that according to the approximate solution in Eqs. (1a) and (1b), at the entrance and exit of the corrugated section, as well as for a smooth heat transfer section, the flow becomes the classical parabolic fully developed velocity profile for parallel-plates, given in the present coordinates system by:

$u^{*}\left(y^{*}\right)=\frac{6}{\left(y_{0}^{*}-y_{1}^{*}\right)^{2}}\left[-y^{* 2}+y^{*}\left(y_{0}^{*}+y_{1}^{*}\right)-y_{0}^{*} y_{1}^{*}\right]$

while the transversal velocity component vanishes in these regions.

Once the velocity field is available, the energy equation in the whole region is given as:

$$
\begin{gathered}
\frac{\partial T^{*}}{\partial t^{*}}+u^{*} \frac{\partial T^{*}}{\partial x^{*}}+v^{*} \frac{\partial T^{*}}{\partial y^{*}}=\alpha_{f}\left(\frac{\partial^{2} T^{*}}{\partial x^{* 2}}+\frac{\partial^{2} T^{*}}{\partial y^{* 2}}\right), \\
0<x^{*}<L^{*}, f y_{0}^{*}\left(x^{*}\right)<y^{*}<f y_{1}^{*}\left(x^{*}\right), t^{*}>0
\end{gathered}
$$

$T^{*}\left(x^{*}, y^{*}, 0\right)=T_{0}^{*}\left(x^{*}, y^{*}\right)$

$T^{*}\left(0, y^{*}, t^{*}\right)=T_{e}^{*}\left(y^{*}, t^{*}\right) ;\left.\quad \frac{\partial T^{*}}{\partial x^{*}}\right|_{x^{*}=L^{*}}=0$

$\left\{\begin{array}{l}\left.\frac{\partial T^{*}}{\partial y^{*}}\right|_{y^{*}=f y_{0}^{*}\left(x^{*}\right)}=0 \\ \left.\frac{\partial T^{*}}{\partial y^{*}}\right|_{y^{*}=f y_{1}^{*}\left(x^{*}\right)}=0\end{array}\right.$

$\left\{\begin{array}{l}T^{*}\left(x^{*}, y^{*}=f y_{0}^{*}\left(x^{*}\right), t^{*}\right)=T_{w, 0}^{*} \\ T^{*}\left(x^{*}, y^{*}=f y_{1}^{*}\left(x^{*}\right), t^{*}\right)=T_{w, 1}^{*}\end{array}, \quad\right.$ for $\quad L_{1}^{*} \leqslant x^{*} \leqslant L_{2}^{*} \quad(3 \mathrm{e}-\mathrm{h})$

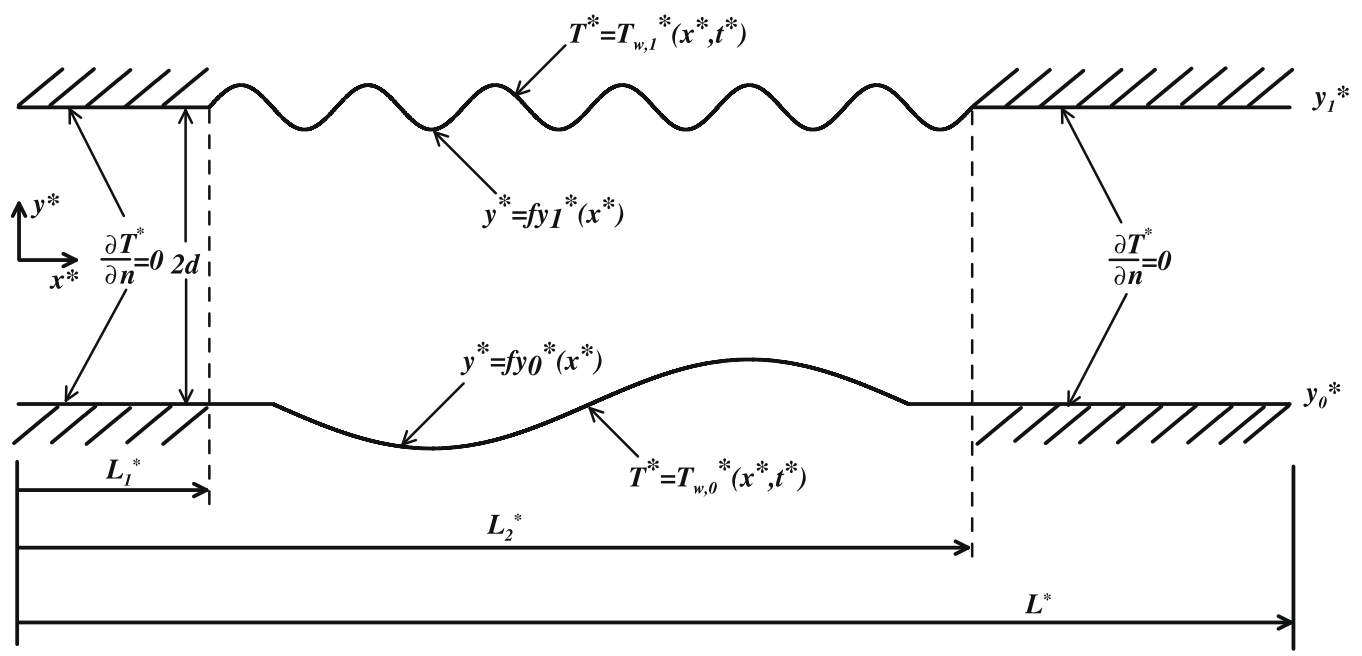

Fig. 1. Geometry and coordinates system for heat transfer in corrugated channel. 
$\left\{\begin{array}{l}\left.\frac{\partial T^{*}}{\partial y^{*}}\right|_{y^{*}=f y_{0}^{*}\left(x^{*}\right)}=0 \\ \left.\frac{\partial T^{*}}{\partial y^{*}}\right|_{y^{*}=f y_{1}^{*}\left(x^{*}\right)}=0\end{array}\right.$, for $L_{2}^{*}<x^{*}<L^{*}$

The following dimensionless groups are defined:

$\xi=\frac{\alpha_{f}}{u_{m} d^{2}} x^{*}=\frac{1}{P e} \frac{x^{*}}{d}, \quad L=\frac{\alpha_{f}}{u_{m} d^{2}} L^{*}=\frac{1}{P e} \frac{L^{*}}{d}$,

$L_{1}=\frac{\alpha_{f}}{u_{m} d^{2}} L_{1}^{*}=\frac{1}{P e} \frac{L_{1}^{*}}{d}, \quad L_{2}=\frac{\alpha_{f}}{u_{m} d^{2}} L_{2}^{*}=\frac{1}{P e} \frac{L_{2}^{*}}{d}$,

$y=\frac{y^{*}}{d}, \quad u(\xi, y)=\frac{u^{*}\left(x^{*}, y^{*}\right)}{u_{m}}, \quad v(\xi, y)=\frac{v^{*}\left(x^{*}, y^{*}\right)}{u_{m}}$,

$t=\frac{\alpha_{f}}{d^{2}} t^{*}, \quad T_{A}(\xi, y, t)=\frac{T^{*}\left(x^{*}, y^{*}, t^{*}\right)-T_{w, 0}^{*}}{\Delta T_{c}}$,

$P e=\frac{u_{m} d}{\alpha_{f}}, \quad d=\frac{y_{1}^{*}-y_{0}^{*}}{2}$

$(4 a-k)$

Then, the dimensionless form of the temperature problem is written as:

$$
\begin{aligned}
\frac{\partial T_{A}}{\partial t} & +u(\xi, y) \frac{\partial T_{A}}{\partial \xi}+\operatorname{Pev}(\xi, y) \frac{\partial T_{A}}{\partial y}=\frac{1}{P e^{2}} \frac{\partial^{2} T_{A}}{\partial \xi^{2}}+\frac{\partial^{2} T_{A}}{\partial y^{2}}, \\
0 & <\xi<L, f y_{0}(\xi)<y<f y_{1}(\xi), t>0
\end{aligned}
$$

$T_{A}(\xi, y, 0)=T_{A, 0}(\xi, y) ; \quad T_{A}(0, y, t)=T_{A, e}(y, t) ;\left.\quad \frac{\partial T_{A}}{\partial \xi}\right|_{\xi=L}=0$

$\left\{\begin{array}{l}\left.\frac{\partial T_{A}}{\partial y}\right|_{y=f y_{0}(\xi)}=0 \\ \left.\frac{\partial T_{A}}{\partial y}\right|_{y=f y_{1}(\xi)}=0\end{array}\right.$

$\left\{\begin{array}{l}T_{A}\left(\xi, y=f y_{0}(\xi), t\right)=0 \\ T_{A}\left(\xi, y=f y_{1}(\xi), t\right)=T_{A, w, 1}\end{array}, \quad\right.$ for $\quad L_{1} \leqslant \xi \leqslant L_{2}$

$\left\{\begin{array}{l}\left.\frac{\partial T_{A}}{\partial y}\right|_{y=f y_{0}(\xi)}=0 \\ \left.\frac{\partial T_{A}}{\partial y}\right|_{y=f y_{1}(\xi)}=0\end{array}\right.$, for $\quad L_{2}<\xi<L$

It can be observed from Eqs. (5g) and ( $5 \mathrm{~h}$ ) that the formulation does not impose symmetry to the thermal problem boundary conditions in the heat transfer section, but if symmetry prevails, Eq. (5h) shall be an homogeneous one.

In light of the discontinuity on the boundary conditions at the wall junction of the three regions, it is more convenient to propose a domain decomposition to handle the three mathematical problems coupled at the cross sections between each pair of regions. The three problems formulation should therefore include the continuity conditions of temperature and heat flux at the fluid interfaces between the regions. Thus, the problem formulation for the first adiabatic region becomes:

$$
\begin{aligned}
& \frac{\partial T_{1}}{\partial t}+u(\xi, y) \frac{\partial T_{1}}{\partial \xi}+\operatorname{Pev}(\xi, y) \frac{\partial T_{1}}{\partial y}=\frac{1}{P e^{2}} \frac{\partial^{2} T_{1}}{\partial \xi^{2}}+\frac{\partial^{2} T_{1}}{\partial y^{2}}, \\
& 0<\xi<L_{1}, f y_{0}(\xi)<y<f y_{1}(\xi), t>0
\end{aligned}
$$

$T_{1}(\xi, y, 0)=T_{A, 0}(\xi, y)$

$T_{1}(0, y, t)=T_{A, e}(y, t) ;\left.\quad \frac{\partial T_{1}}{\partial \xi}\right|_{\xi=L_{1}}=\left.\frac{\partial T_{A, 2}}{\partial \xi}\right|_{\xi=L_{1}} ;$

$\left.\frac{\partial T_{1}}{\partial y}\right|_{y=f y_{0}(\xi)}=0 ;\left.\quad \frac{\partial T_{1}}{\partial y}\right|_{y=f y_{1}(\xi)}=0$
For the heat exchanging section, we have:

$$
\begin{aligned}
& \frac{\partial T_{A, 2}}{\partial t}+u(\xi, y) \frac{\partial T_{A, 2}}{\partial \xi}+\operatorname{Pev}(\xi, y) \frac{\partial T_{A, 2}}{\partial y} \\
& \quad=\frac{1}{P e^{2}} \frac{\partial^{2} T_{A, 2}}{\partial \xi^{2}}+\frac{\partial^{2} T_{A, 2}}{\partial y^{2}}, \quad L_{1}<\xi<L_{2}, f y_{0}(\xi)<y<f y_{1}(\xi), \\
& t>0 \\
& T_{A, 2}(\xi, y, 0)=T_{A, 0}(\xi, y) \\
& T_{A, 2}\left(\xi=L_{1}, y, t\right)=T_{1}\left(\xi=L_{1}, y, t\right) ; \\
& T_{A, 2}\left(\xi=L_{2}, y, t\right)=T_{3}\left(\xi=L_{2}, y, t\right) \\
& T_{A, 2}\left(\xi, y=f y_{0}(\xi), t\right)=0 ; \quad T_{A, 2}\left(\xi, y=f y_{1}(\xi), t\right)=T_{A, w, 1}
\end{aligned}
$$

and finally for the exiting section, also adiabatic, we may write:

$\frac{\partial T_{3}}{\partial t}+u(\xi, y) \frac{\partial T_{3}}{\partial \xi}+\operatorname{Pev}(\xi, y) \frac{\partial T_{3}}{\partial y}=\frac{1}{P e^{2}} \frac{\partial^{2} T_{3}}{\partial \xi^{2}}+\frac{\partial^{2} T_{3}}{\partial y^{2}}$,

$$
L_{2}<\xi<L, f y_{0}(\xi)<y<f y_{1}(\xi), t>0
$$

$T_{3}(\xi, y, 0)=T_{A, 0}(\xi, y)$

$\left.\frac{\partial T_{3}}{\partial \xi}\right|_{\xi=L_{2}}=\left.\frac{\partial T_{A, 2}}{\partial \xi}\right|_{\xi=L_{2}} ;\left.\quad \frac{\partial T_{3}}{\partial \xi}\right|_{\xi=L}=0 ;\left.\quad \frac{\partial T_{3}}{\partial y}\right|_{y=f y_{0}(\xi)}=0 ;$
$\left.\frac{\partial T_{3}}{\partial y}\right|_{y=f y_{1}(\xi)}=0$

The temperature problem for the heated section remains nonhomogeneous in Eq. (7f). In order to homogenize the problem in the transversal direction $y$, a filtering solution, $\phi(\xi, y)$, is employed for the potential $T_{A, 2}(\xi, y, t)$, in the form:

$T_{A, 2}(\xi, y, t)=T_{2}(\xi, y, t)+\phi(\xi, y)$

A simple and sufficiently general form for the filter $\phi(\xi, y)$ is obtained by satisfying the diffusion operator in the transversal direction, which yields:

$\phi(\xi, y)=T_{A, w, 1} \frac{f y_{0}(\xi)-y}{f y_{0}(\xi)-f y_{1}(\xi)}$

Then, the homogeneous problem for the corrugated section is given by:

$\frac{\partial T_{2}}{\partial t}+u(\xi, y) \frac{\partial T_{2}}{\partial \xi}+\operatorname{Pev}(\xi, y) \frac{\partial T_{2}}{\partial y}$
$\quad=\frac{1}{P e^{2}} \frac{\partial^{2} T_{2}}{\partial \xi^{2}}+\frac{\partial^{2} T_{2}}{\partial y^{2}}+g(\xi, y), \quad L_{1}<\xi<L_{2}$,

$f y_{0}(\xi)<y<f y_{1}(\xi), t>0$

$T_{2}(\xi, y, t=0)=T_{A, 0}(\xi, y)-\phi(\xi, y)$

$T_{2}\left(\xi=L_{1}, y, t\right)=T_{1}\left(\xi=L_{1}, y, t\right)-\phi\left(\xi=L_{1}, y\right) ;$

$T_{2}\left(\xi=L_{2}, y, t\right)=T_{3}\left(\xi=L_{2}, y, t\right)-\phi\left(\xi=L_{2}, y\right)$

$T_{2}\left(\xi, y=f y_{0}(\xi), t\right)=0 ; \quad T_{2}\left(\xi, y=f y_{1}(\xi), t\right)=0$

where

$g(\xi, y)=\frac{1}{P e^{2}} \frac{\partial^{2} \phi}{\partial \xi^{2}}-\operatorname{Pev}(\xi, y) \frac{\partial \phi}{\partial y}-u(\xi, y) \frac{\partial \phi}{\partial \xi}$

Next, the decomposed domain is described with three different coordinates systems, which match at the two interfaces, as shown in Fig. 2 below. The normalized longitudinal coordinates are then computed in terms of the original dimensionless coordinate as: 


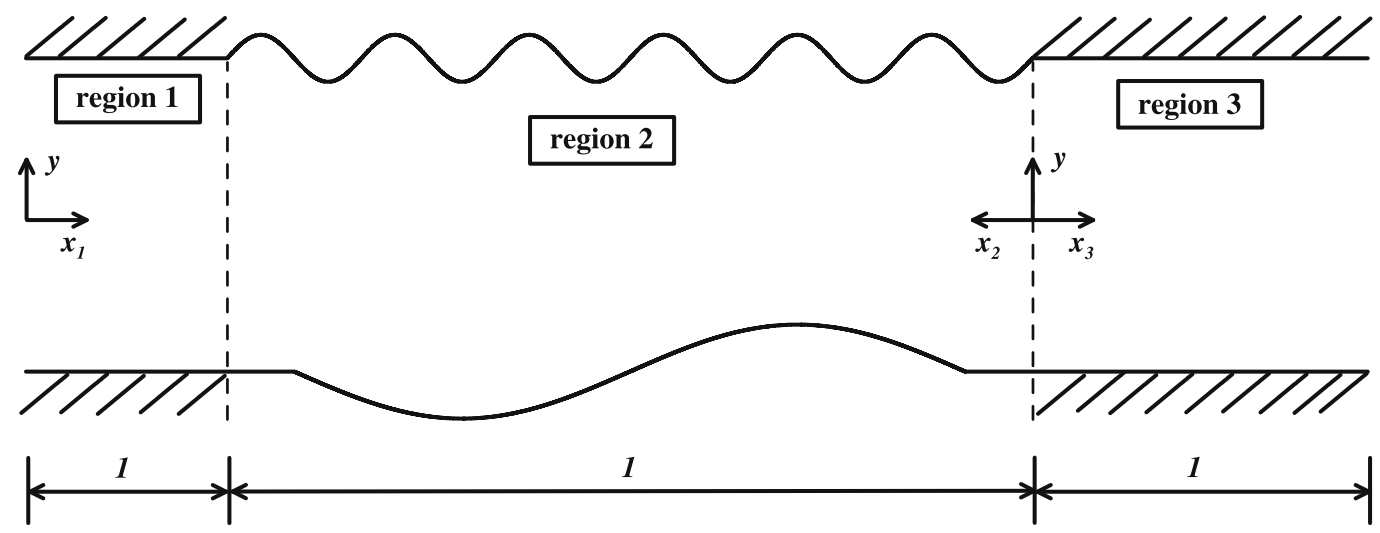

Fig. 2. Coordinates systems for the decomposed domain.

$x_{1}=\frac{1}{L_{1}} \xi ; \quad x_{2}=\frac{1}{\left(L_{2}-L_{1}\right)}\left(L_{2}-\xi\right) ; \quad x_{3}=\frac{1}{\left(L-L_{2}\right)}\left(\xi-L_{2}\right)$

Thus, in terms of the redefined coordinates systems the dimensionless problem formulation is given, for each of the three regions, as:

$$
\begin{aligned}
& \frac{\partial T_{1}}{\partial t}+u\left(x_{1}, y\right) \frac{\partial T_{1}}{\partial x_{1}} \frac{1}{L_{1}}+\operatorname{Pev}\left(x_{1}, y\right) \frac{\partial T_{1}}{\partial y} \\
& =\frac{1}{P e^{2}} \frac{1}{L_{1}^{2}} \frac{\partial^{2} T_{1}}{\partial x_{1}^{2}}+\frac{\partial^{2} T_{1}}{\partial y^{2}}, \quad 0<x_{1}<1, y_{0}<y<y_{1}, t>0 \\
& T_{1}\left(x_{1}, y, t=0\right)=T_{1,0}\left(x_{1}, y\right) \\
& T_{1}\left(x_{1}=0, y, t\right)=T_{1, e}(y, t) ;\left.\quad \frac{\partial T_{1}}{\partial x_{1}}\right|_{x_{1}=1}=-\left.\frac{L_{1}}{L_{2}-L_{1}} \frac{\partial T_{2}}{\partial x_{2}}\right|_{x_{2}=1} \\
& \left.\frac{\partial T_{1}}{\partial y}\right|_{y=y_{0}}=0 ;\left.\quad \frac{\partial T_{1}}{\partial y}\right|_{y=y_{1}}=0 \\
& \frac{\partial T_{2}}{\partial t}-\frac{1}{\left(L_{2}-L_{1}\right)} u\left(x_{2}, y\right) \frac{\partial T_{2}}{\partial x_{2}}+\operatorname{Pev}\left(x_{2}, y\right) \frac{\partial T_{2}}{\partial y} \\
& =\frac{1}{P e^{2}} \frac{1}{\left(L_{2}-L_{1}\right)^{2}} \frac{\partial^{2} T_{2}}{\partial x_{2}^{2}}+\frac{\partial^{2} T_{2}}{\partial y^{2}}+g\left(x_{2}, y\right), \\
& 0<x_{2}<1, f y_{0}\left(x_{2}\right)<y<f y_{1}\left(x_{2}\right), t>0 \\
& T_{2}\left(x_{2}, y, t=0\right)=T_{2,0}\left(x_{2}, y\right)-\phi\left(x_{2}, y\right) \\
& T_{2}\left(x_{2}=0, y, t\right)=T_{3}\left(x_{3}=0, y, t\right)-\phi\left(x_{2}=0, y\right) ; \\
& T_{2}\left(x_{2}=1, y, t\right)=T_{1}\left(x_{1}=1, y, t\right)-\phi\left(x_{2}=1, y\right) \\
& T_{2}\left(x_{2}, y=f y_{0}\left(x_{2}\right), t\right)=0 ; \quad T_{2}\left(x_{2}, y=f y_{1}\left(x_{2}\right), t\right)=0 \\
& \phi\left(x_{2}, y\right)=T_{A, w, 1} \frac{f y_{0}\left(x_{2}\right)-y}{f y_{0}\left(x_{2}\right)-f y_{1}\left(x_{2}\right)} ; \\
& g\left(x_{2}, y\right)=\frac{1}{P e^{2}\left(L_{2}-L_{1}\right)^{2}} \frac{\partial^{2} \phi}{\partial x_{2}^{2}}-\operatorname{Pev}\left(x_{2}, y\right) \frac{\partial \phi}{\partial y} \\
& +\frac{1}{L_{2}-L_{1}} u\left(x_{2}, y\right) \frac{\partial \phi}{\partial x_{2}} \\
& \frac{\partial T_{3}}{\partial t}+\frac{1}{\left(L-L_{2}\right)} u\left(x_{3}, y\right) \frac{\partial T_{3}}{\partial x_{3}}+\operatorname{Pev}\left(x_{3}, y\right) \frac{\partial T_{3}}{\partial y} \\
& =\frac{1}{P e^{2}} \frac{1}{\left(L-L_{2}\right)^{2}} \frac{\partial^{2} T_{3}}{\partial x_{3}^{2}}+\frac{\partial^{2} T_{3}}{\partial y^{2}}, \quad 0<x_{3}<1, \\
& y_{0}<y<y_{1}, t>0
\end{aligned}
$$

$T_{3}\left(x_{3}, y, t=0\right)=T_{3,0}\left(x_{3}, y\right)$

$\left.\frac{\partial T_{3}}{\partial x_{3}}\right|_{x_{3}=0}=-\left.\frac{L-L_{2}}{L_{2}-L_{1}} \frac{\partial T_{2}}{\partial x_{2}}\right|_{x_{2}=0} ;\left.\quad \frac{\partial T_{3}}{\partial x_{3}}\right|_{x_{3}=1}=0$

$\left.\frac{\partial T_{3}}{\partial y}\right|_{y=y_{0}}=0 ;\left.\quad \frac{\partial T_{3}}{\partial y}\right|_{y=y_{1}}=0$

One may observe that after the coordinates systems redefinition, the problems are coupled only at the interfaces and all the domain limits are the same, and therefore the dependent variables may be algebraically interpreted as applied to one single domain in the longitudinal coordinate $(0<\mathrm{x}<1)$.

Following the formalism in the GITT [7-9], the auxiliary problems are now defined to construct the eigenfunction expansions in each region. For regions 1 and 3 we adopt the same eigenvalue problem, with second kind boundary conditions, given by:

$\frac{d^{2} \psi_{i}}{d y^{2}}+\mu_{i}^{2} \psi_{i}(y)=0, \quad y_{0}<y<y_{1}$

$\left.\frac{d \psi_{i}}{d y}\right|_{y=y_{0}}=0 ;\left.\quad \frac{d \psi_{i}}{d y}\right|_{y=y_{1}}=0$

whose solution in terms of eigenfunctions, norms and eigenvalues is readily found as

$$
\begin{aligned}
& \psi_{i}(y)= \begin{cases}1, & i=0 \\
\cos \left[\mu_{i}\left(y-y_{0}\right)\right], & i=1,2,3 \ldots\end{cases} \\
& N \psi_{i}=\int_{y_{0}}^{y_{1}} \psi_{i}^{2}(y) d y=\left\{\begin{array}{ll}
y_{1}-y_{0}, & i=0 \\
\frac{y_{1}-y_{0}}{2}, & i=1,2,3 \ldots
\end{array} ;\right. \\
& \mu_{i}= \begin{cases}0, & i=0 \\
\frac{i \pi}{y_{1}-y_{0}}, & i=1,2,3 \ldots\end{cases}
\end{aligned}
$$

For region 2, the auxiliary problem has to account for the irregular walls, which is incorporated into the eigenfunctions and eigenvalues via the functions of $x$ that describe the transversal domains, as shown below:

$$
\begin{aligned}
& \frac{\partial^{2} \Gamma_{m}}{\partial y^{2}}+\beta_{m}^{2}(x) \Gamma_{m}(x, y)=0, \quad f y_{0}(x)<y<f y_{1}(x) \\
& \Gamma_{m}\left[y=f y_{0}(x)\right]=0 ; \quad \Gamma_{m}\left[y=f y_{1}(x)\right]=0
\end{aligned}
$$


Thus, the $x$-dependent eigenfunctions, norms and eigenvalues are given by:

$\Gamma_{m}(x, y)=\sin \left\{\beta_{m}(x)\left[y-f y_{0}(x)\right]\right\}$

$N \Gamma_{m}(x)=\int_{f y_{0}(x)}^{f y_{1}(x)} \Gamma_{m}^{2}(x, y) d y=\frac{f y_{1}(x)-f y_{0}(x)}{2}$

$\beta_{m}(x)=\frac{m \pi}{f y_{1}(x)-f y_{0}(x)}, \quad m=1,2,3 \ldots$

$(17 \mathrm{~d}-\mathrm{f})$

Once the eigenvalue problems have been defined and solved, the integral transform pairs (transform-inverse) are constructed as:

$\bar{T}_{1, i}(x, t)=\int_{y_{0}}^{y_{1}} T_{1}(x, y, t) \psi_{i}(y) d y ;$

$T_{1}(x, y, t)=\sum_{i=0}^{\infty} \frac{1}{N \psi_{i}} \bar{T}_{1, i}(x, t) \psi_{i}(y)$

$\bar{T}_{2, m}(x, t)=\int_{f y_{0}(x)}^{f y_{1}(x)} T_{2}(x, y, t) \Gamma_{m}(x, y) d y ;$

$T_{2}(x, y, t)=\sum_{m=1}^{\infty} \frac{1}{N \Gamma_{m}(x)} \bar{T}_{2, m}(x, t) \Gamma_{m}(x, y)$

(19a, b)

$\bar{T}_{3, i}(x, t)=\int_{y_{0}}^{y_{1}} T_{3}(x, y, t) \psi_{i}(y) d y$

$T_{3}(x, y, t)=\sum_{i=0}^{\infty} \frac{1}{N \psi_{i}} \bar{T}_{3, i}(x, t) \psi_{i}(y)$

$(20 \mathrm{a}, \mathrm{b})$

One may start with the integral transformation of Eqs. (13) for region 1 , operating with $\int_{y_{0}}^{y_{1}} \bullet \psi_{i}(y) d y$, and after substitution of the inverse formula (18b) and some manipulation, the transformed system becomes:

$\frac{\partial \bar{T}_{1, i}}{\partial t}+\frac{1}{L_{1}} \sum_{j=0}^{\infty} A_{i j} \frac{\partial \bar{T}_{1, j}}{\partial x}=\frac{1}{P e^{2} L_{1}^{2}} \frac{\partial^{2} \bar{T}_{1, i}}{\partial x^{2}}-\mu_{i}^{2} \bar{T}_{1, i}(x, t)$,
$0<x<1, t>0$

$\bar{T}_{1, i}(x, t=0)=\bar{T}_{1,0, i}(x)$

$\bar{T}_{1, i}(x=0, t)=\bar{T}_{e, i}(t)$

$\left.\frac{\partial \bar{T}_{1, i}}{\partial x}\right|_{x=1}=-\left.\frac{L_{1}}{L_{2}-L_{1}} \sum_{n=1}^{\infty} \frac{1}{N \Gamma_{n}(x=1)} B_{i n}(x=1) \frac{\partial \bar{T}_{2, n}}{\partial x}\right|_{x=1}$

Similarly, Eqs. (14) are operated on with $\int_{f y_{0}(x)}^{f y_{1}(x)} \bullet \Gamma_{m}(x, y) d y$ to yield the transformed version for the temperature problem in region 2:

$$
\begin{aligned}
& \frac{\partial \bar{T}_{2, m}}{\partial t}=\frac{1}{P e^{2}\left(L_{2}-L_{1}\right)^{2}} \frac{\partial^{2} \bar{T}_{2, m}}{\partial x^{2}}+\sum_{n=1}^{\infty} \frac{\partial \bar{T}_{2, n}}{\partial x} \\
& \quad \times\left[\frac{1}{P e^{2}\left(L_{2}-L_{1}\right)^{2}}\left(2 \frac{d}{d x}\left(\frac{1}{N \Gamma_{n}(x)}\right) N \Gamma_{n}(x) \delta_{m n}+\frac{2}{N \Gamma_{n}(x)} F_{m n}(x)\right)\right. \\
& \left.\quad+\frac{1}{L_{2}-L_{1}}\left(\frac{1}{N \Gamma_{n}(x)} D_{m n}(x)\right)\right]+\sum_{n=1}^{\infty} \bar{T}_{2, n}(x, t) \\
& \quad \times\left[\frac { 1 } { P e ^ { 2 } ( L _ { 2 } - L _ { 1 } ) ^ { 2 } } \left(\frac{d^{2}}{d x^{2}}\left(\frac{1}{N \Gamma_{n}(x)}\right) N \Gamma_{n}(x) \delta_{m n}+2 \frac{d}{d x}\left(\frac{1}{N \Gamma_{n}(x)}\right)\right.\right. \\
& \left.\quad \times F_{m n}(x)+\frac{1}{N \Gamma_{n}(x)} G_{m n}(x)\right)+\frac{1}{L_{2}-L_{1}} \\
& \quad \times\left(\frac{1}{N \Gamma_{n}(x)} C_{m n}(x)+\frac{d}{d x}\left(\frac{1}{N \Gamma_{n}(x)}\right) D_{m n}(x)\right) \\
& \left.-P e\left(\frac{1}{N \Gamma_{n}(x)} E_{m n}(x)\right)-\beta_{m}^{2}(x) \delta_{m n}\right]+\bar{g}_{m}(x), \quad 0<x<1, t>0
\end{aligned}
$$

$$
\bar{T}_{2, m}(x, t=0)=\bar{T}_{2,0, m}(x)-\bar{\phi}_{m}(x)
$$

$$
\bar{T}_{2, m}(x=0, t)=\sum_{j=0}^{\infty}\left(\frac{1}{N \psi_{j}} B_{m j}(x=0) \bar{T}_{3, j}(x=0, t)\right)-\bar{\phi}_{m}(x=0)
$$

$$
\bar{T}_{2, m}(x=1, t)=\sum_{j=0}^{\infty}\left(\frac{1}{N \psi_{j}} B_{m j}(x=1) \bar{T}_{1, j}(x=1, t)\right)-\bar{\phi}_{m}(x=1)
$$

And finally, for region 3, Eqs. (15) are operated on with $\int_{y_{0}}^{y_{1}} \bullet \psi_{i}(y) d y$, to furnish:

$$
\begin{aligned}
& \frac{\partial \bar{T}_{3, i}}{\partial t}+\frac{1}{L-L_{2}} \sum_{j=0}^{\infty} A_{i j} \frac{\partial \bar{T}_{3, j}}{\partial x}=\frac{1}{P e^{2}\left(L-L_{2}\right)^{2}} \frac{\partial^{2} \bar{T}_{3, i}}{\partial x^{2}} \\
& \quad-\mu_{i}^{2} \bar{T}_{3, i}(x, t), \quad 0<x<1, t>0 \\
& \bar{T}_{3, i}(x, t=0)=\bar{T}_{3,0, i}(x)
\end{aligned}
$$

$$
\begin{aligned}
& \left.\frac{\partial \bar{T}_{3, i}}{\partial x}\right|_{x=0}=-\left.\frac{L-L_{2}}{L_{2}-L_{1}} \sum_{n=1}^{\infty} \frac{1}{N \Gamma_{n}(x=0)} B_{i n}(x=0) \frac{\partial \bar{T}_{2, n}}{\partial x}\right|_{x=0} ; \\
& \left.\frac{\partial \bar{T}_{3, i}}{\partial x}\right|_{x=1}=0
\end{aligned}
$$

The coefficients that appear on the transformed system Eqs. (21)-(23) are analytically obtained from the following integrations:

$A_{i j}=\frac{1}{N \psi_{j}} \int_{y_{0}}^{y_{1}} u(y) \psi_{i}(y) \psi_{j}(y) d y$

$B_{n i}(x)=\int_{y_{0}}^{y_{1}} \psi_{i}(y) \Gamma_{n}(x, y) d y$

$$
\begin{aligned}
& C_{m n}(x)=\int_{f y_{0}(x)}^{f y_{1}(x)} u(x, y) \Gamma_{m}(x, y) \frac{\partial \Gamma_{n}}{\partial x} d y \\
& D_{m n}(x)=\int_{f y_{0}(x)}^{f y_{1}(x)} u(x, y) \Gamma_{m}(x, y) \Gamma_{n}(x, y) d y
\end{aligned}
$$

$E_{m n}(x)=\int_{f y_{0}(x)}^{f y_{1}(x)} v(x, y) \Gamma_{m}(x, y) \frac{\partial \Gamma_{n}}{\partial y} d y$

$F_{m n}(x)=\int_{f y_{0}(x)}^{f y_{1}(x)} \Gamma_{m}(x, y) \frac{\partial \Gamma_{n}}{\partial x} d y$

$G_{m n}(x)=\int_{f y_{0}(x)}^{f y_{1}(x)} \Gamma_{m}(x, y) \frac{\partial^{2} \Gamma_{n}}{\partial x^{2}} d y$

$\bar{g}_{m}(x)=\int_{f y_{0}(x)}^{f y_{1}(x)} g(x, y) \Gamma_{m}(x, y) d y$;

$\bar{\phi}_{m}(x)=\int_{f y_{0}(x)}^{f y_{1}(x)} \phi(x, y) \Gamma_{m}(x, y) d y$

Eqs. (21)-(23) form an infinite system of partial differential equations having as independent variables the unified dimensionless longitudinal coordinate, $x$, and dimensionless time, $t$, and as dependent variables the transformed temperatures in each region. For computational purposes, the system is truncated to a finite order, truncating the eigenfunction expansions for each field in a sufficiently large number of terms for each region $\left(N_{1}, N_{2}\right.$ and $\left.N_{3}\right)$. Due to the $x$-variable nature of the system coefficients, Eqs. (24), the PDE system has to be numerically solved, for instance employ- 
ing the Method of Lines with local error control as implemented on the function NDSolve of the Mathematica symbolic-numerical computation platform [10].

For the thermal problem, results are reported in terms of the bulk temperature and the local Nusselt numbers at the two channel walls (yielding the same results for the symmetric situation), as defined below:

$T_{m}(\xi, t)=\frac{\int_{f y_{0}(\xi)}^{f y_{1}(\xi)} u(\xi, y) T_{A}(\xi, y, t) d y}{\int_{f y_{0}(\xi)}^{f y_{1}(\xi)} u(\xi, y) d y}$

$N u_{1}(\xi, t)=\frac{-4}{T_{m}(\xi, t)}\left(\nabla T_{A} \cdot \mathbf{n}_{y_{1}}\right)$

$N u_{0}(\xi, t)=\frac{4}{T_{m}(\xi, t)}\left(\nabla T_{A} \cdot \mathbf{n}_{y_{0}}\right)$

In the chosen example discussed below, the problem formulation adopts the same geometry and boundary conditions as presented by Wang and Chen [18]. The walls boundaries are then described by the following functions along the longitudinal coordinate which describe a symmetric sinusoidal wavy profile:

$f y_{0}^{*}\left(x^{*}\right)= \begin{cases}y_{0}^{*}, & 0<x^{*}<L_{1}^{*} \\ y_{0}^{*}+\alpha^{*} \sin \left(\pi\left(x^{*}-L_{1}^{*}\right) \frac{12}{L_{2}^{*}}\right), & L_{1}^{*}<x^{*}<L_{2}^{*} \\ y_{0}^{*}, & L_{2}^{*}<x^{*}<L^{*}\end{cases}$
$f y_{1}^{*}\left(x^{*}\right)= \begin{cases}y_{1}^{*}, & 0<x^{*}<L_{1}^{*} \\ y_{1}^{*}-\alpha^{*} \sin \left(\pi\left(x^{*}-L_{1}^{*}\right) \frac{12}{L_{2}^{*}}\right), & L_{1}^{*}<x^{*}<L_{2}^{*} \\ y_{1}^{*}, & L_{2}^{*}<x^{*}<L^{*}\end{cases}$

\section{Results and discussion}

A computational code was developed and implemented on the Mathematica system [10] and the transformed partial differential Eqs. (21)-(23) were solved by employing the subroutine NDSolve, which uses the numerical Method of Lines to handle a wide range of coupled PDEs. Numerical results were produced for bulk temperature and local Nusselt numbers in both situations of smooth and wavy channels. First, a brief convergence analysis of the temperature field is presented and a few comparisons with literature results for simpler models are provided for validation purposes. Second, a physical interpretation of the axial conduction effects in smooth ducts is offered, in light of the possible low values of the Peclet number, followed by the relevant parametric analysis in terms of the Reynolds number and the wavy wall geometry effects on heat transfer enhancement.

The convergence behavior of the proposed eigenfunction expansion for the temperature fields is analyzed in terms of the following parameters: truncation orders of the expansions for each region $\left(N_{1}, N_{2}, N_{3}\right)$; maximum step size along the spatial coordinate during the numerical solution of the transformed system (MaxStepSize parameter of the NDSolve subroutine [10]). Such

Table 1

Dimensionless lengths of the sub-domains for each Peclet number used in the simulations.

\begin{tabular}{llll}
\hline Pe & \multicolumn{3}{l}{ Dimensionless lengths } \\
\cline { 2 - 4 } & $L_{1}$ & $L_{2}$ & $\mathrm{~L}$ \\
\hline 1 & 200 & 230 & 280 \\
10 & 5 & 10 & 15 \\
30 & 1 & 5 & 6 \\
100 & 0.15 & 4.15 & 4.45 \\
\hline
\end{tabular}

parameter is indirectly controlled via the minimum number of steps in the domain discretization allowed for in the algorithm (parameter MS, which stands for Max Steps).

The values of Peclet number adopted in the present analysis were $P e=1,10,30$ and 100 . The dimensionless longitudinal lengths used in the simulations for each Peclet number are listed in Table 1 below, which have proven to be compatible with the physical problem here formulated, i.e., they are sufficiently large so as to warrant satisfaction of the prescribed boundary conditions at the inlet and outlet of the channel.

The constructed computer code was first verified against the thermal problem results for transient convection within smooth parallel-plates channels $[13,14]$ with one single region, i.e., just the heat transfer section. A thorough convergence analysis was then undertaken on the bulk and centerline temperature results

Table 2

Convergence behavior of the centerline temperature as function of the dimensionless time for $P e=30$ along the three sub-regions of a smooth channel with different truncation orders $N_{1}$ and $N_{2}=8, N_{3}=4$ and $\mathrm{MS}=50$.

\begin{tabular}{|c|c|c|c|c|c|}
\hline$t$ & $\xi$ & $N_{1}=2$ & $N_{1}=4$ & $N_{1}=6$ & $N_{1}=8$ \\
\hline \multicolumn{6}{|c|}{ Centerline temperature } \\
\hline \multirow[t]{9}{*}{0.1} & 0.05 & 1.00186 & 0.99669 & 0.99815 & 0.99771 \\
\hline & 0.09 & 0.99858 & 0.98834 & 0.99064 & 0.98999 \\
\hline & 0.13 & 0.98290 & 0.96775 & 0.97071 & 0.96988 \\
\hline & 0.17 & 0.94467 & 0.92650 & 0.92975 & 0.92884 \\
\hline & 0.21 & 0.87445 & 0.85637 & 0.85943 & 0.85857 \\
\hline & 0.25 & 0.76784 & 0.75289 & 0.75534 & 0.75462 \\
\hline & 0.29 & 0.62938 & 0.61934 & 0.62100 & 0.62046 \\
\hline & 0.33 & 0.47374 & 0.46877 & 0.46967 & 0.46930 \\
\hline & 0.37 & 0.32224 & 0.32120 & 0.32158 & 0.32134 \\
\hline \multirow[t]{10}{*}{0.5} & 0.05 & 1.00002 & 0.99962 & 0.99974 & 0.99970 \\
\hline & 0.14 & 0.99881 & 0.99672 & 0.99715 & 0.99702 \\
\hline & 0.23 & 0.98762 & 0.98218 & 0.98316 & 0.98287 \\
\hline & 0.32 & 0.94645 & 0.93767 & 0.93925 & 0.93880 \\
\hline & 0.41 & 0.85043 & 0.84088 & 0.84280 & 0.84226 \\
\hline & 0.50 & 0.68889 & 0.68140 & 0.68316 & 0.68266 \\
\hline & 0.59 & 0.48152 & 0.47710 & 0.47828 & 0.47792 \\
\hline & 0.68 & 0.27631 & 0.27454 & 0.27511 & 0.27490 \\
\hline & 0.77 & 0.12341 & 0.12326 & 0.12347 & 0.12339 \\
\hline & 0.86 & 0.04082 & 0.04118 & 0.04124 & 0.04123 \\
\hline \multirow[t]{10}{*}{1.0} & 0.10 & 0.99985 & 0.99984 & 0.99984 & 0.99984 \\
\hline & 0.26 & 0.99964 & 0.99943 & 0.99946 & 0.99945 \\
\hline & 0.42 & 0.99664 & 0.99547 & 0.99562 & 0.99557 \\
\hline & 0.58 & 0.97813 & 0.97480 & 0.97526 & 0.97510 \\
\hline & 0.74 & 0.91262 & 0.90717 & 0.90809 & 0.90780 \\
\hline & 0.90 & 0.76446 & 0.75879 & 0.76010 & 0.75972 \\
\hline & 1.06 & 0.52500 & 0.52262 & 0.52223 & 0.52308 \\
\hline & 1.22 & 0.26621 & 0.26700 & 0.26704 & 0.26714 \\
\hline & 1.38 & 0.10090 & 0.10103 & 0.10104 & 0.10107 \\
\hline & 1.54 & 0.02708 & 0.02702 & 0.02703 & 0.02704 \\
\hline \multirow[t]{9}{*}{1.5} & 0.30 & 0.99985 & 0.99984 & 0.99984 & 0.99984 \\
\hline & 0.52 & 0.99962 & 0.99949 & 0.99950 & 0.99950 \\
\hline & 0.74 & 0.99654 & 0.99573 & 0.99581 & 0.99577 \\
\hline & 0.96 & 0.97918 & 0.96987 & 0.97210 & 0.97164 \\
\hline & 1.18 & 0.75643 & 0.75519 & 0.75517 & 0.75562 \\
\hline & 1.40 & 0.45739 & 0.45761 & 0.45764 & 0.45788 \\
\hline & 1.62 & 0.24305 & 0.24358 & 0.24361 & 0.24372 \\
\hline & 1.84 & 0.10587 & 0.10622 & 0.10623 & 0.10627 \\
\hline & 2.06 & 0.03380 & 0.03389 & 0.03389 & 0.03390 \\
\hline \multirow[t]{10}{*}{1.9} & 0.20 & 0.99985 & 0.99985 & 0.99985 & 0.99985 \\
\hline & 0.48 & 0.99985 & 0.99984 & 0.99984 & 0.99984 \\
\hline & 0.76 & 0.99962 & 0.99951 & 0.99952 & 0.99952 \\
\hline & 1.04 & 0.96859 & 0.95545 & 0.95366 & 0.95729 \\
\hline & 1.32 & 0.63269 & 0.63222 & 0.63220 & 0.63264 \\
\hline & 1.60 & 0.36454 & 0.36431 & 0.36430 & 0.36453 \\
\hline & 1.88 & 0.19564 & 0.19567 & 0.19568 & 0.19579 \\
\hline & 2.16 & 0.09006 & 0.09023 & 0.09024 & 0.09028 \\
\hline & 2.44 & 0.03111 & 0.03120 & 0.03121 & 0.03122 \\
\hline & 2.72 & 0.00679 & 0.00680 & 0.00680 & 0.00680 \\
\hline
\end{tabular}


Table 3

Convergence behavior of the bulk temperature as function of the dimensionless time for $P e=30$ along the three sub-regions of a smooth channel with different truncation orders $N_{1}$ and $N_{2}=8, N_{3}=4$ and $\mathrm{MS}=50$.

\begin{tabular}{|c|c|c|c|c|c|}
\hline$t$ & $\xi$ & $N_{1}=2$ & $N_{1}=4$ & $N_{1}=6$ & $N_{1}=8$ \\
\hline \multicolumn{6}{|c|}{ Bulk temperature } \\
\hline \multirow[t]{9}{*}{0.1} & 0.05 & 0.98772 & 0.98774 & 0.98779 & 0.98779 \\
\hline & 0.09 & 0.96227 & 0.96278 & 0.96289 & 0.96291 \\
\hline & 0.13 & 0.91567 & 0.91735 & 0.91755 & 0.91758 \\
\hline & 0.17 & 0.84351 & 0.84663 & 0.84689 & 0.84694 \\
\hline & 0.21 & 0.74526 & 0.74919 & 0.74947 & 0.74952 \\
\hline & 0.25 & 0.62488 & 0.62838 & 0.62860 & 0.62865 \\
\hline & 0.29 & 0.49100 & 0.49303 & 0.49314 & 0.49317 \\
\hline & 0.33 & 0.35628 & 0.35667 & 0.35668 & 0.35668 \\
\hline & 0.37 & 0.23501 & 0.23438 & 0.23434 & 0.23433 \\
\hline \multirow[t]{10}{*}{0.5} & 0.05 & 0.99902 & 0.99892 & 0.99892 & 0.99892 \\
\hline & 0.14 & 0.99139 & 0.99096 & 0.99094 & 0.99094 \\
\hline & 0.23 & 0.96276 & 0.96240 & 0.96242 & 0.96242 \\
\hline & 0.32 & 0.89243 & 0.89335 & 0.89345 & 0.89347 \\
\hline & 0.41 & 0.76603 & 0.76870 & 0.76889 & 0.76892 \\
\hline & 0.50 & 0.58916 & 0.59222 & 0.59242 & 0.59245 \\
\hline & 0.59 & 0.39093 & 0.39261 & 0.39273 & 0.39274 \\
\hline & 0.68 & 0.21385 & 0.21397 & 0.21402 & 0.21401 \\
\hline & 0.77 & 0.09167 & 0.09130 & 0.09132 & 0.09133 \\
\hline & 0.86 & 0.02933 & 0.02913 & 0.02915 & 0.02916 \\
\hline \multirow[t]{10}{*}{1.0} & 0.10 & 0.99982 & 0.99981 & 0.99981 & 0.99981 \\
\hline & 0.26 & 0.99903 & 0.99889 & 0.99888 & 0.99888 \\
\hline & 0.42 & 0.99243 & 0.99193 & 0.99190 & 0.99190 \\
\hline & 0.58 & 0.96205 & 0.96136 & 0.96135 & 0.96134 \\
\hline & 0.74 & 0.87438 & 0.87460 & 0.87465 & 0.87465 \\
\hline & 0.90 & 0.70359 & 0.70537 & 0.70550 & 0.70555 \\
\hline & 1.06 & 0.41799 & 0.41997 & 0.42005 & 0.42021 \\
\hline & 1.22 & 0.19611 & 0.19680 & 0.19683 & 0.19690 \\
\hline & 1.38 & 0.07252 & 0.07260 & 0.07261 & 0.07263 \\
\hline & 1.54 & 0.01904 & 0.01899 & 0.01900 & 0.01901 \\
\hline \multirow[t]{9}{*}{1.5} & 0.30 & 0.99983 & 0.99982 & 0.99982 & 0.99982 \\
\hline & 0.52 & 0.99929 & 0.99919 & 0.99918 & 0.99918 \\
\hline & 0.74 & 0.99381 & 0.99338 & 0.99336 & 0.99335 \\
\hline & 0.96 & 0.95015 & 0.94894 & 0.94903 & 0.94961 \\
\hline & 1.18 & 0.58119 & 0.58104 & 0.58104 & 0.58139 \\
\hline & 1.40 & 0.34425 & 0.34446 & 0.34448 & 0.34466 \\
\hline & 1.62 & 0.18146 & 0.18187 & 0.18189 & 0.18198 \\
\hline & 1.84 & 0.07810 & 0.07836 & 0.07837 & 0.07840 \\
\hline & 2.06 & 0.02452 & 0.02458 & 0.02458 & 0.02459 \\
\hline \multirow[t]{10}{*}{1.9} & 0.20 & 0.99985 & 0.99985 & 0.99985 & 0.99985 \\
\hline & 0.48 & 0.99984 & 0.99983 & 0.99983 & 0.99983 \\
\hline & 0.76 & 0.99936 & 0.99927 & 0.99927 & 0.99927 \\
\hline & 1.04 & 0.83701 & 0.83763 & 0.83759 & 0.83819 \\
\hline & 1.32 & 0.48041 & 0.48011 & 0.48010 & 0.48043 \\
\hline & 1.60 & 0.27577 & 0.27560 & 0.27560 & 0.27577 \\
\hline & 1.88 & 0.14738 & 0.14741 & 0.14742 & 0.14750 \\
\hline & 2.16 & 0.06729 & 0.06742 & 0.06743 & 0.06746 \\
\hline & 2.44 & 0.02293 & 0.02300 & 0.02300 & 0.02301 \\
\hline & 2.72 & 0.00491 & 0.00491 & 0.00491 & 0.00492 \\
\hline
\end{tabular}

so as to provide confidence on the numerical results to be reported. For instance, Tables 2 and 3 bring the convergence behavior of the centerline and bulk temperatures, respectively, for $P e=30$ in the case of a smooth walls duct. Clearly, the expansions convergence rates improve with both increasing dimensionless time and longitudinal coordinate, as expected, within the range of these independent variables as covered in Tables 2 and 3 . In synthesis, the adoption of the truncation orders $N_{1}=8, N_{2}=8$ and $N_{3}=4$ was sufficient to provide three to four converged significant digits in the temperature field throughout these tabulated results, in a fairly wide range of dimensionless time and longitudinal coordinate and for the proposed values of Peclet number. It was also observed, though not evident from the single $P e$ number tables here presented, that the truncation order of the expansions is required to gradually increase as $P e$ increases.
Table 4

Convergence behavior of the centerline temperature as function of the dimensionless time for $P e=30$ along the three sub-regions of a corrugated channel $(\alpha=0.1)$ with different values of the sub-domains discretization parameter MS and $N_{1}=4, N_{2}=8$ and $N_{3}=4$

\begin{tabular}{|c|c|c|c|c|}
\hline$t$ & $\xi$ & $\mathrm{MS}=100$ & $\mathrm{MS}=150$ & $\mathrm{MS}=200$ \\
\hline \multicolumn{5}{|c|}{ Centerline temperature } \\
\hline \multirow[t]{6}{*}{0.1} & 0.05 & 0.96101 & 0.96103 & 0.96102 \\
\hline & 0.08 & 0.88657 & 0.88662 & 0.88661 \\
\hline & 0.11 & 0.74938 & 0.74940 & 0.74939 \\
\hline & 0.14 & 0.55846 & 0.55842 & 0.55842 \\
\hline & 0.17 & 0.35510 & 0.35501 & 0.35502 \\
\hline & 0.20 & 0.18778 & 0.18778 & 0.18780 \\
\hline \multirow[t]{10}{*}{0.5} & 0.05 & 0.99962 & 0.99962 & 0.99962 \\
\hline & 0.14 & 0.99672 & 0.99672 & 0.99672 \\
\hline & 0.23 & 0.98218 & 0.98218 & 0.98218 \\
\hline & 0.32 & 0.93768 & 0.93767 & 0.93767 \\
\hline & 0.41 & 0.84089 & 0.84088 & 0.84088 \\
\hline & 0.50 & 0.68140 & 0.68139 & 0.68139 \\
\hline & 0.59 & 0.47707 & 0.47708 & 0.47708 \\
\hline & 0.68 & 0.27451 & 0.27454 & 0.27455 \\
\hline & 0.77 & 0.12328 & 0.12330 & 0.12330 \\
\hline & 0.86 & 0.04121 & 0.04121 & 0.04120 \\
\hline \multirow[t]{10}{*}{1.0} & 0.10 & 0.99984 & 0.99984 & 0.99984 \\
\hline & 0.26 & 0.99943 & 0.99943 & 0.99943 \\
\hline & 0.42 & 0.99547 & 0.99547 & 0.99547 \\
\hline & 0.58 & 0.97480 & 0.97480 & 0.97480 \\
\hline & 0.74 & 0.90717 & 0.90716 & 0.90716 \\
\hline & 0.90 & 0.75880 & 0.75879 & 0.75878 \\
\hline & 1.06 & 0.56627 & 0.56578 & 0.56567 \\
\hline & 1.22 & 0.33640 & 0.33612 & 0.33604 \\
\hline & 1.38 & 0.09140 & 0.09139 & 0.09137 \\
\hline & 1.54 & 0.01859 & 0.01857 & 0.01857 \\
\hline \multirow[t]{9}{*}{1.5} & 0.30 & 0.99984 & 0.99984 & 0.99984 \\
\hline & 0.52 & 0.99949 & 0.99949 & 0.99949 \\
\hline & 0.74 & 0.99573 & 0.99573 & 0.99573 \\
\hline & 0.96 & 0.97002 & 0.96971 & 0.96960 \\
\hline & 1.18 & 0.90198 & 0.90066 & 0.90034 \\
\hline & 1.40 & 0.33787 & 0.33746 & 0.33735 \\
\hline & 1.62 & 0.15549 & 0.15529 & 0.15524 \\
\hline & 1.84 & 0.10100 & 0.10089 & 0.10085 \\
\hline & 2.06 & 0.02252 & 0.02250 & 0.02249 \\
\hline \multirow[t]{10}{*}{1.9} & 0.20 & 0.99985 & 0.99985 & 0.99985 \\
\hline & 0.48 & 0.99984 & 0.99984 & 0.99984 \\
\hline & 0.76 & 0.99951 & 0.99951 & 0.99951 \\
\hline & 1.04 & 0.99679 & 0.99667 & 0.99639 \\
\hline & 1.32 & 0.59252 & 0.59161 & 0.59137 \\
\hline & 1.60 & 0.21974 & 0.21939 & 0.21930 \\
\hline & 1.88 & 0.17429 & 0.17401 & 0.17395 \\
\hline & 2.16 & 0.04220 & 0.04214 & 0.04213 \\
\hline & 2.44 & 0.02116 & 0.02114 & 0.02113 \\
\hline & 2.72 & 0.00370 & 0.00369 & 0.00369 \\
\hline
\end{tabular}

In the sequence, Tables 4 and 5 illustrate the convergence behavior of the centerline and bulk temperatures, respectively, for the wavy channel case, with $P e=30$ and $\alpha=0.1$, by varying the sub-domains discretization parameter MS (maximum number of steps) and with $N_{1}=4, N_{2}=8$ and $N_{3}=4$. Here the objective is to illustrate the effect of demanding more refined grids from the Method of Lines numerical solution, and it has been verified that the results are apparently converged to at least four significant digits with MS $=150$. Thus, this particular combination of parameters values was utilized for all subsequent simulations.

Next, an attempt was made of validating the present analysis, for the smooth parallel-plates case, against a previously reported analytical solution [19] that accounts for the upstream adiabatic region, under steady laminar forced convection for low Peclet numbers. Fig. 3 thus presents such comparison in terms of the dimensionless bulk temperature, where only the behavior along 
Table 5

Convergence behavior of the bulk temperature as function of the dimensionless time for $P e=30$ along the three sub-regions of a corrugated channel $(\alpha=0.1)$ with different values of the sub-domains discretization parameter MS and $N_{1}=4, N_{2}=8$ and $N_{3}=4$.

\begin{tabular}{|c|c|c|c|c|}
\hline$t$ & $\xi$ & $\mathrm{MS}=100$ & $\mathrm{MS}=150$ & $\mathrm{MS}=200$ \\
\hline \multicolumn{5}{|c|}{ Bulk temperature } \\
\hline \multirow[t]{6}{*}{0.1} & 0.05 & 0.90490 & 0.90492 & 0.90491 \\
\hline & 0.08 & 0.78181 & 0.78184 & 0.78183 \\
\hline & 0.11 & 0.61260 & 0.61260 & 0.61260 \\
\hline & 0.14 & 0.42326 & 0.42322 & 0.42322 \\
\hline & 0.17 & 0.25115 & 0.25109 & 0.25111 \\
\hline & 0.20 & 0.12509 & 0.12509 & 0.12510 \\
\hline \multirow[t]{10}{*}{0.5} & 0.05 & 0.99892 & 0.99892 & 0.99892 \\
\hline & 0.14 & 0.99096 & 0.99096 & 0.99096 \\
\hline & 0.23 & 0.96241 & 0.96240 & 0.96240 \\
\hline & 0.32 & 0.89335 & 0.89335 & 0.89335 \\
\hline & 0.41 & 0.76870 & 0.76870 & 0.76870 \\
\hline & 0.50 & 0.59221 & 0.59221 & 0.59221 \\
\hline & 0.59 & 0.39259 & 0.39260 & 0.39260 \\
\hline & 0.68 & 0.21396 & 0.21397 & 0.21398 \\
\hline & 0.77 & 0.09132 & 0.09132 & 0.09133 \\
\hline & 0.86 & 0.02916 & 0.02915 & 0.02915 \\
\hline \multirow[t]{10}{*}{1.0} & 0.10 & 0.99981 & 0.99981 & 0.99981 \\
\hline & 0.26 & 0.99889 & 0.99889 & 0.99889 \\
\hline & 0.42 & 0.99193 & 0.99193 & 0.99193 \\
\hline & 0.58 & 0.96137 & 0.96136 & 0.96136 \\
\hline & 0.74 & 0.87460 & 0.87460 & 0.87460 \\
\hline & 0.90 & 0.70543 & 0.70539 & 0.70537 \\
\hline & 1.06 & 0.49158 & 0.49103 & 0.49086 \\
\hline & 1.22 & 0.24372 & 0.24354 & 0.24348 \\
\hline & 1.38 & 0.05385 & 0.05383 & 0.05383 \\
\hline & 1.54 & 0.01254 & 0.01253 & 0.01252 \\
\hline \multirow[t]{9}{*}{1.5} & 0.30 & 0.99982 & 0.99982 & 0.99982 \\
\hline & 0.52 & 0.99919 & 0.99919 & 0.99919 \\
\hline & 0.74 & 0.99338 & 0.99338 & 0.99338 \\
\hline & 0.96 & 0.95029 & 0.94923 & 0.94882 \\
\hline & 1.18 & 0.71454 & 0.71342 & 0.71314 \\
\hline & 1.40 & 0.20636 & 0.20609 & 0.20602 \\
\hline & 1.62 & 0.12681 & 0.12666 & 0.12661 \\
\hline & 1.84 & 0.07906 & 0.07897 & 0.07894 \\
\hline & 2.06 & 0.01336 & 0.01335 & 0.01335 \\
\hline \multirow[t]{10}{*}{1.9} & 0.20 & 0.99985 & 0.99985 & 0.99985 \\
\hline & 0.48 & 0.99983 & 0.99983 & 0.99983 \\
\hline & 0.76 & 0.99927 & 0.99927 & 0.99927 \\
\hline & 1.04 & 0.91993 & 0.91767 & 0.91708 \\
\hline & 1.32 & 0.37605 & 0.37549 & 0.37534 \\
\hline & 1.60 & 0.17770 & 0.17741 & 0.17734 \\
\hline & 1.88 & 0.13025 & 0.13004 & 0.12999 \\
\hline & 2.16 & 0.02830 & 0.02826 & 0.02825 \\
\hline & 2.44 & 0.01768 & 0.01766 & 0.01765 \\
\hline & 2.72 & 0.00219 & 0.00219 & 0.00218 \\
\hline
\end{tabular}

the heat transfer section is presented. Different values of a modified Peclet number are employed, here called $P e_{T}$, according to the definition in [19]. A fairly good adherence between the two independent solutions can be observed providing further validation to the proposed hybrid methodology. Also, one may already observe the marked influence of the axial diffusion along the fluid on the temperature behavior within the actual heat transfer section.

The influence of the axial heat diffusion on the channel transient thermal response is now more closely examined and Fig. 4a and $b$ show a comparison of the transient behavior of the bulk temperature in a smooth channel for the cases of $P e=1$ and 30 and at different dimensionless times. One must observe that the longitudinal coordinate was multiplied by the Peclet number in these graphs, so that the resulting variable is essentially the channel length normalized by the transversal dimension. Clearly, the lower Pe number case $(P e=1)$ involves a much more significant partici-

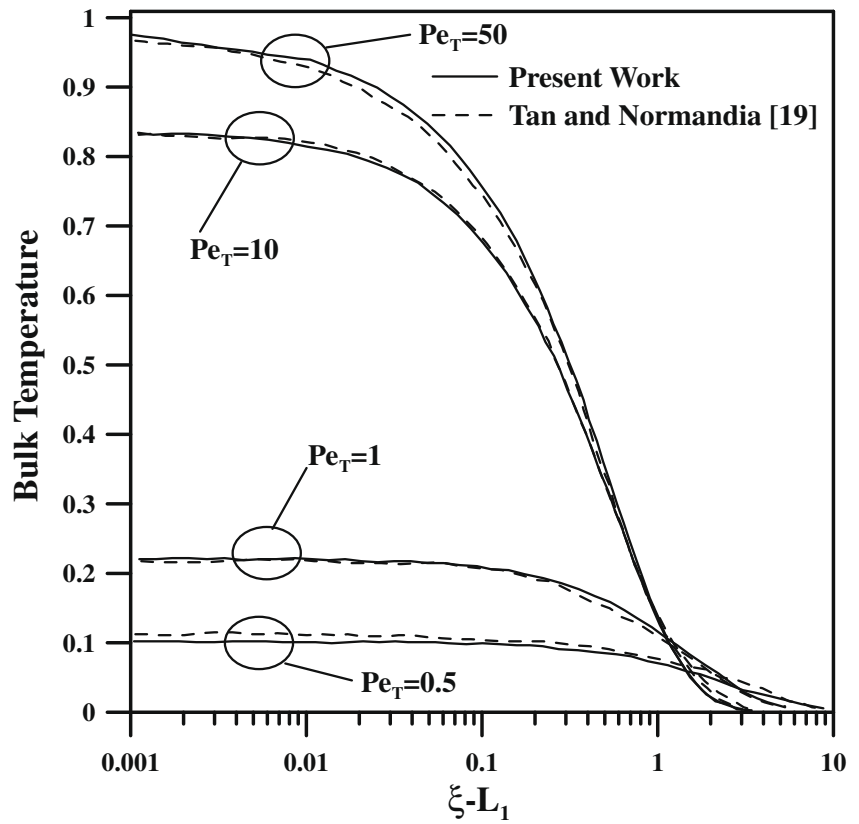

Fig. 3. Comparison of dimensionless bulk temperatures from present GITT solution and from analytical steady state solution in [19] for a smooth parallel-plates channel with an upstream adiabatic region and low Peclet number.

pation of the upstream region in the channel thermal behavior, within a longer transient process as well. For the higher value of $P e=30$, the participating upstream region is quite reduced in comparison with the previous case, while in terms of dimensionless time the steady state is achieved much faster.

Fig. 5 then illustrates the influence of the axial heat diffusion within the fluid for the steady state dimensionless bulk temperatures along the channel for $P e=1,10$, and 30 , and especially across the interface between the upstream adiabatic region and the heat transfer section. The pre-cooling (or pre-heating) effect provided by the presence of the upstream region is quite noticeable for the lower values of Peclet number $(P e=1$ and 10$)$, which is quite relevant along the transient state as well, as shown in Fig. 4. As a result, a heat transfer enhancement effect is in fact observed, resulting in lower temperature values within the same physical region, for decreasing Peclet number. As can be deduced, significant errors may occur if experimental results are employed to estimate Nusselt numbers that assume the inlet temperature at the heat transfer section inlet as having the same value as the uniform temperature at the upstream region inlet, as usually considered for ordinary liquids at the macro-scale.

The next step in the present analysis is the illustration of the adequacy of the approximate velocity field, Eqs. (1), in describing the flow behavior within the wavy channel, as shown below in Fig. 6. As previously mentioned, the simplified approach is expected to provide reasonable results for lower values of the Reynolds number and for smoother corrugations. Thus, numerical results for the full Navier-Stokes formulation were employed in the verification of the present approximate analytical solutions, as obtained from the GITT hybrid numerical-analytical solution available in [17]. For instance, Fig. 6a presents the longitudinal velocity component at different axial positions along the corrugated section, for the chosen values of $R e=10$ and $\alpha=0.1$, while Fig. $6 \mathrm{~b}$ and $\mathrm{c}$ present the longitudinal velocity component for progressively less smooth corrugations, with $\alpha=0.2$ and $\alpha=0.3$, respectively. It can be noticed that the increase in wall corrugation amplitude leads to a less accurate velocity component as obtained from the approximate relations of Eqs. (1), especially in regions closer to the inlet of the wavy region. Also, increasing the Reynolds 

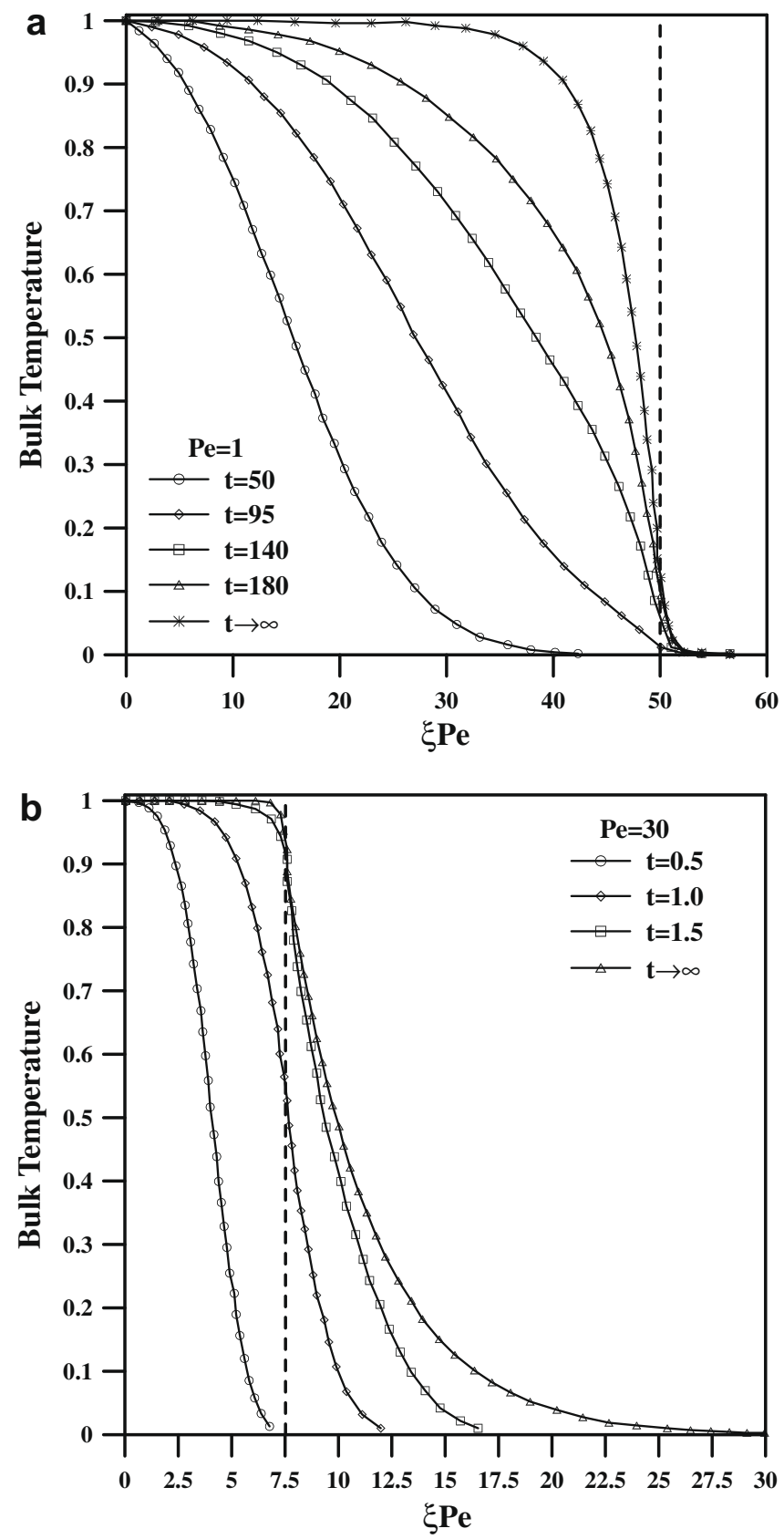

Fig. 4. Transient behavior of the dimensionless bulk temperature in smooth parallel-plates channel along both the upstream adiabatic region and the heat transfer section (dashed line is the interface between the two regions): (a) $P e=1$; (b) $P e=30$.

number leads to some loss of accuracy in the simplified solution, but one can see from Fig. $6 \mathrm{~d}$ below that the results are still reasonably accurate for the case of $R e=100$ and $\alpha=0.1$, and apparently the increase in corrugation amplitude of Fig. $6 \mathrm{~b}$ and $\mathrm{c}$ was more significant in deviating the approximate solution from the converged GITT results for the full Navier-Stokes formulation than the increase in Reynolds number of Fig. 6d. The comparisons of the longitudinal velocity component are emphasized since the related convective term is the most important one in the heat transfer problem to be addressed.

Fig. 7 illustrates the transient behavior of the bulk temperature in the wavy channel for $P e=10$ and $\alpha=0.1$, where the dashed line denotes the interface between the adiabatic region 1 and the corrugated section, region 2 . Clearly, we may see that due to the

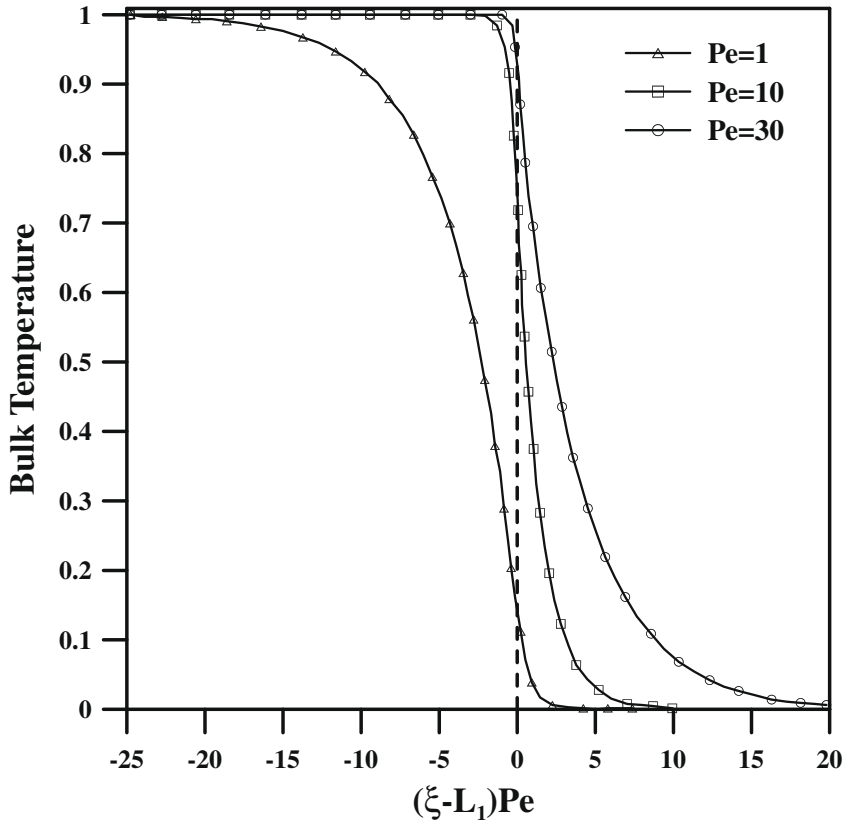

Fig. 5. Comparison of dimensionless bulk temperatures in smooth parallel-plates channel along both the upstream adiabatic region and heat transfer section at steady state for different values of Peclet number (dashed line is the interface between the two regions).

low Peclet number, the axial diffusion along the fluid promotes a sensible effect on the bulk temperature evolution within the access region (region 1 ) along the transient period. The steady state results are however closer to the situation of an unheated inlet section, which would be obtained by the model that neglects axial diffusion of heat within the fluid. Therefore, for micro-channel applications that involve low Peclet numbers, the behavior of the thermal wave front can be markedly affected by the presence of an adiabatic inlet section. It can also be observed that the bulk temperature behavior downstream already presents a slight fluctuating shape due to the presence of the wall corrugations, though the lower values of Peclet tend to smear our the oscillations.

Next, Fig. 8 illustrates the effect of the Peclet number on the bulk temperatures temperature in the wavy channel with $\alpha=0.1$ for the steady state situation, by taking the two values $P e=10$ and 30 . Similarly to the smooth channel situation, one may clearly observe the more significant participation of region 1 in the thermal exchange due to the lower value of Pe, but also the more pronounced effects on the bulk temperature fluctuations due to the wall corrugations in the case of a smaller axial diffusion of heat $(P e=30)$, when the transversal effects start playing a major role with respect to the longitudinal conduction.

For the heat transfer enhancement analysis it is of interest to evaluate the behavior of the Nusselt number under different corrugation conditions. Fig. 9, for instance, illustrates the local Nusselt number results for $P e=10$ and $\alpha=0.1$ and 0.2 . The smooth parallel-plates case is also plotted for reference purposes, as the solid black line. One may see that even with the lower corrugation amplitude value some noticeable heat transfer enhancement is already evident, and marked increases in the local heat transfer coefficient are achieved for the higher corrugation amplitude value for this value of $P e$.

\section{Conclusions}

Laminar forced convection in low Reynolds number flows within channels with smooth and corrugated walls was analyzed and 

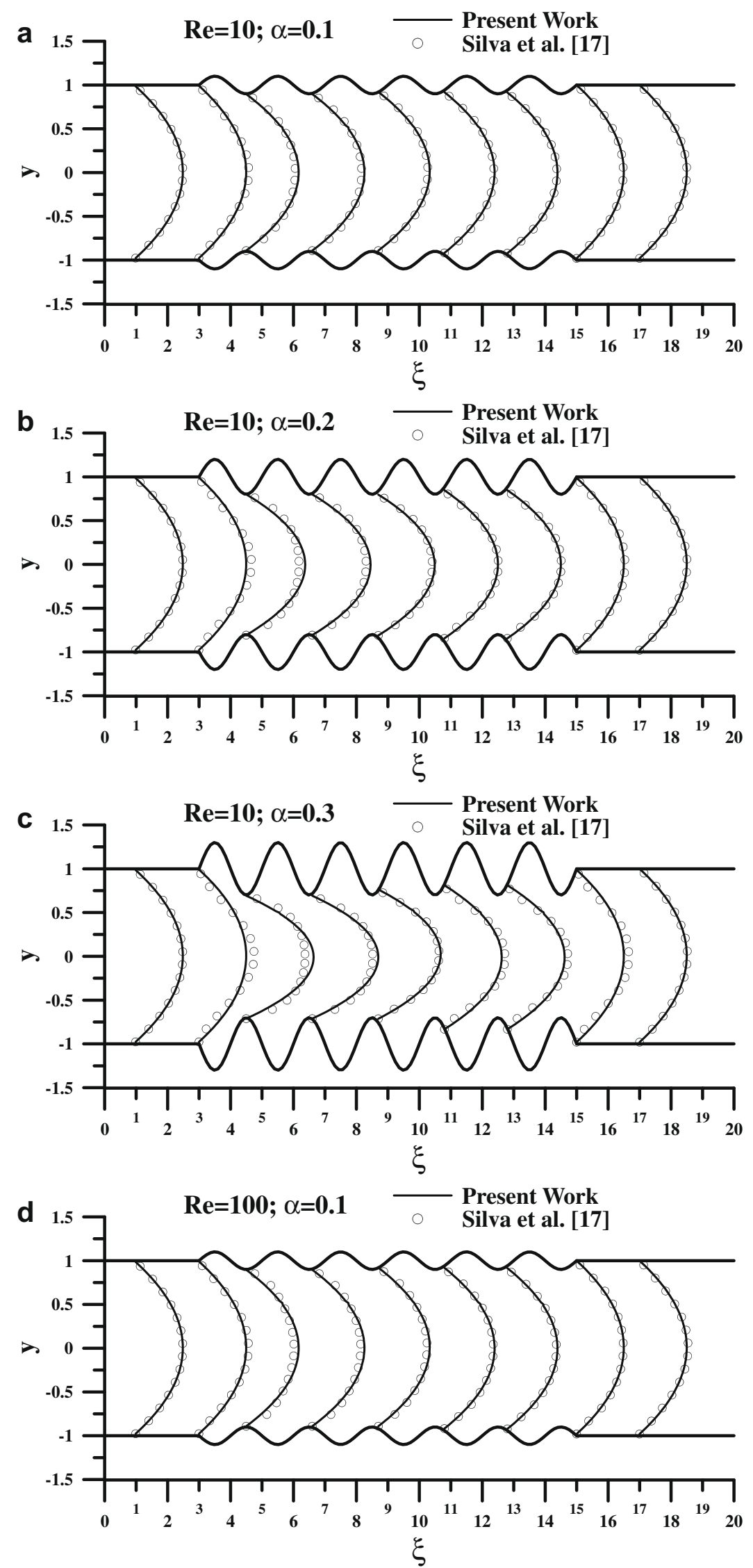

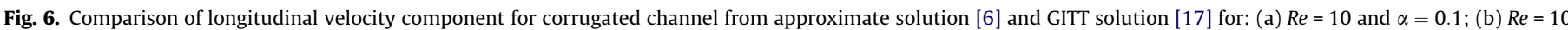
and $\alpha=0.2$; (c) $R e=10$ and $\alpha=0.3$; (d) $R e=100$ and $\alpha=0.1$. 


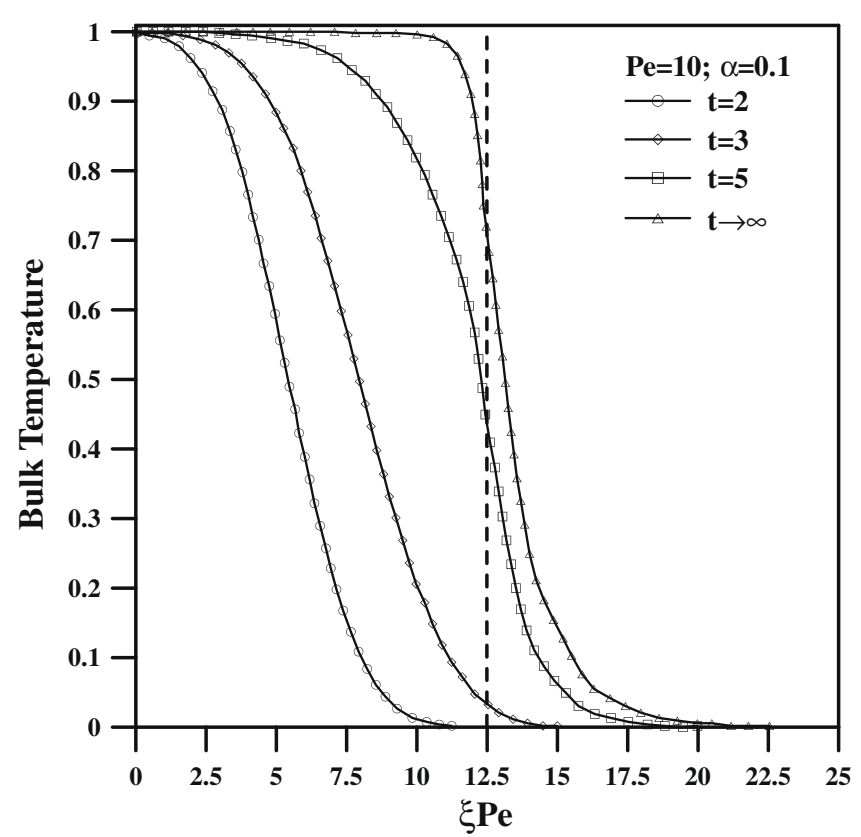

Fig. 7. Transient behavior of bulk temperature for corrugated channel on regions 1 and $2, P e=10, \alpha=0.1$.

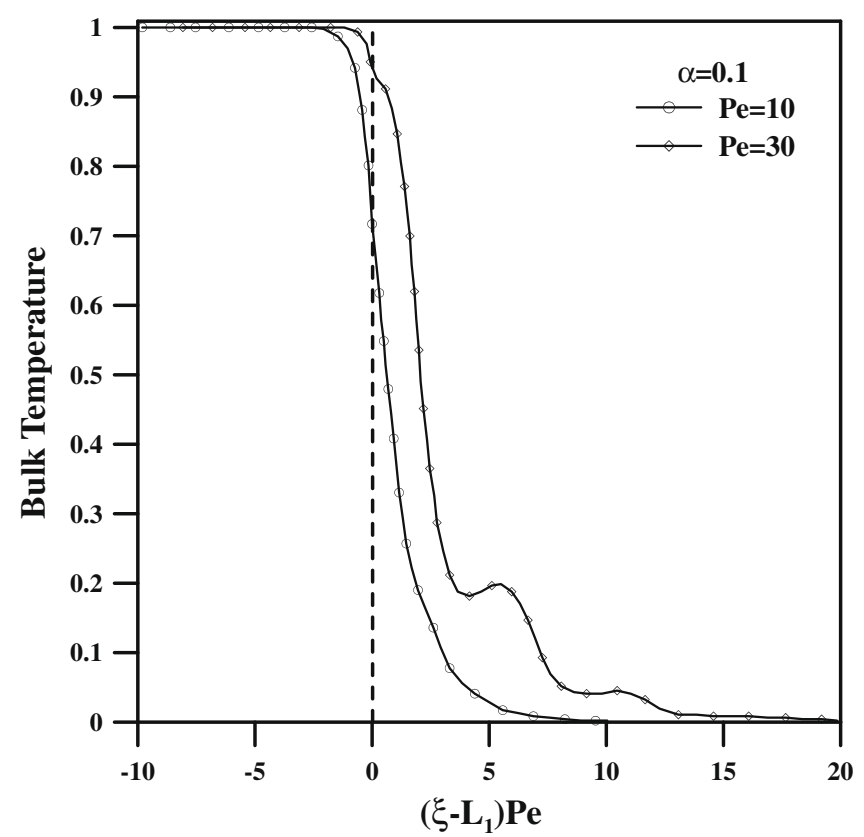

Fig. 8. Influence of Peclet number on the bulk temperature behavior for corrugated channel along regions 1 and 2 in steady state, $P e=10$ and 30 and $\alpha=0.1$.

discussed to investigate possible heat transfer enhancement effects. The physical modeling for the thermal problem includes discontinuities in the boundary conditions at the channel walls, considering the interfaces between entrance and exit adiabatic regions and an intermediate heat transfer section. The thermal problem was then solved with the Generalized Integral Transform Technique (GITT) applied in partial transformation mode and with a transient formulation. The presented results exploited the bulk temperature field and the local Nusselt number within the heat transfer section of the channel. The axial diffusion effect along the fluid was first examined for a smooth parallel-plates channel,

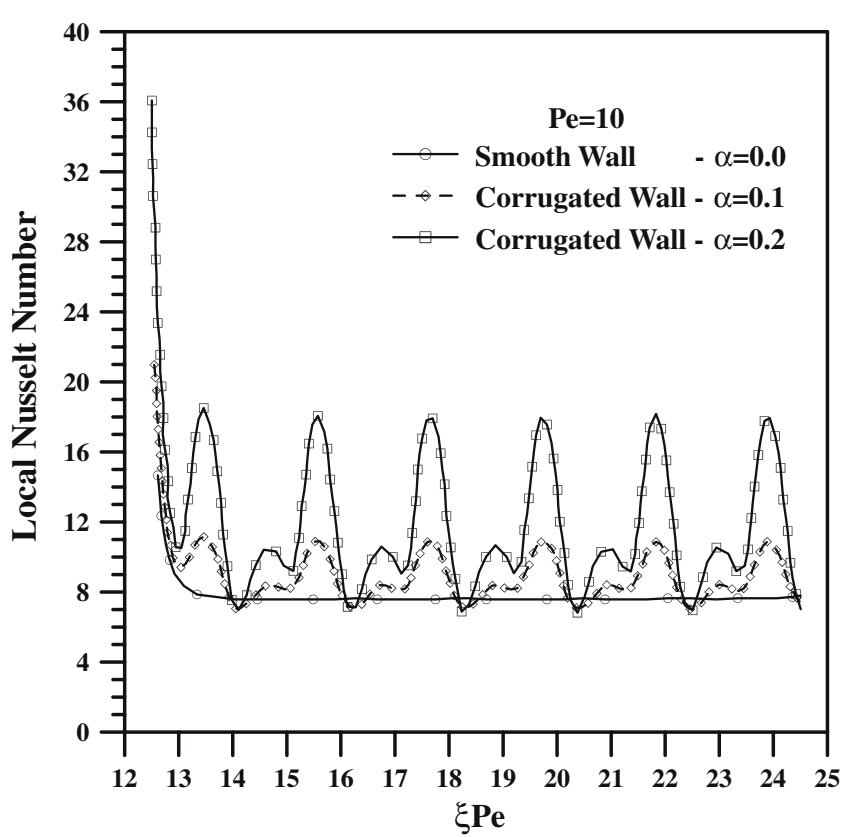

Fig. 9. Local Nusselt numbers at steady state for smooth and corrugated channels with $P e=10$ and $\alpha=0$ (parallel-plates), 0.1 and 0.2 .

in light of the lower values of Peclet number achievable in such applications due to the expectable low values of Reynolds number. The importance of accounting for an upstream adiabatic region was discussed, illustrating the resulting marked changes on the temperature distribution behavior within the actual heat transfer section. Then, for the corrugated heat transfer section case, the velocity field was obtained by making use of an approximate solution methodology, shown to be appropriate for small scale corrugations and low Reynolds numbers. From such results, one may notice the combined influence on the heat transfer enhancement along the heat transfer section due to both the low values of Peclet number and the presence of corrugated walls. The analysis may now proceed towards the utilization of optimization schemes aimed at the identification of wall profiles for optimally enhanced heat transfer, which can then be reconfirmed by the more accurate solution of the flow problem employing the GITT itself, as previously accomplished in [17].

\section{Acknowledgements}

The authors would like to acknowledge the partial financial support provided by CNPq and CENPES/Petrobras S.A., both in Brazil, along the developments here reported.

\section{References}

[1] R.L. Webb, N.H. Kim, Principles of Enhanced Heat Transfer, second ed., Taylor \& Francis, New York, 2005.

[2] Y. Yener, S. Kakaç, M. Avelino, T. Okutucu, Single-phase forced convection in micro-channels - a state-of-the-art review, in: S. Kakaç, L.L. Vasiliev, Y. Bayazitoglu, Y. Yener (Eds.), Microscale Heat Transfer - Fundamentals and Applications NATO ASI Series, Kulwer Academic Publishers, The Netherlands, 2005, pp. 1-24.

[3] M.E. Steinke, S.G. Kandlikar, Single-phase heat transfer enhancement techniques in micro-channel and mini-channel flows, in: Proceedings of the International Conference on Micro-channels and Mini-channels - ASME, Rochester, NY, 2004.

[4] M. Vasudevaiah, K. Balamurugan, Heat transfer of rarefied gases in a corrugated micro-channel, Int. J. Therm. Sci. 40 (2001) 454-468.

[5] C.K. Chen, C.C. Cho, Electrokinetically-driven flow mixing in micro-channels with wavy surface, J. Colloid Interf. Sci. 312 (2007) 470-480.

[6] M.N. Özisik, C. Trepp, H. Egolf, Laminar forced convection in converging or diverging planar symmetric ducts, Int. J. Heat Mass Transfer 25 (1982) 1477-1480. 
[7] R.M. Cotta, Integral Transforms in Computational Heat and Fluid Flow, CRC Press, Boca Raton, 1993.

[8] R.M. Cotta, The Integral Transforms Method in Thermal and Fluids Sciences and Engineering, Begell House, New York, 1998.

[9] R.M. Cotta, M.D. Mikhailov, Hybrid methods and symbolic computations, in: W.J. Minkowycz, E.M. Sparrow, J.Y. Murthy (Eds.), Handbook of Numerical Heat Transfer, second ed., Wiley, New York, 2006, pp. 493-522.

[10] S. Wolfram, The Mathematica Book, version 5.2, Cambridge-Wolfram Media, 2005

[11] R.M. Cotta, J.E.V. Gerk, Mixed finite-difference/integral transform approach for parabolic-hyperbolic problems in transient forced convection, Numer. Heat Transfer, B 25 (1994) 433-448.

[12] R.M. Cotta, S. Kakaç, M.D. Mikhailov, F.V. Castellões, C.R. Cardoso, Transient flow and thermal analysis in microfluidics, in: S. Kakaç, L.L. Vasiliev, Y. Bayazitoglu, Y. Yener (Eds.), Microscale Heat Transfer - Fundamentals and Applications, NATO ASI Series, Kluwer Academic Publishers, The Netherlands, 2005, pp. 175-196.
[13] F.V. Castellões, R.M. Cotta, Analysis of transient and periodic convection in micro-channels via integral transforms, Progr. Comput. Fluid Dynam. 6 (2006) 321-326.

[14] F.V. Castellões, C.R. Cardoso, P. Couto, R.M. Cotta, Transient analysis of slip flow and heat transfer in microchannels, Heat Transfer Eng. 28 (2007) 549-558.

[15] S. Yu, T.A. Ameel, Slip flow heat transfer in rectangular microchannels, Int. J. Heat Mass Transfer 44 (2001) 4225-4234.

[16] G. Tunc, Y. Bayazitoglu, Heat transfer in rectangular microchannels, Int. J. Heat Mass Transfer 45 (2002) 765-773.

[17] R.L. Silva, J.N.N. Quaresma, C.A.C. Santos, R.M., Cotta, Hybrid solution for developing laminar flow in wavy-wall channels via integral transforms, in: Proceedings of the 2007 ASME International Mechanical Engineering Congress \& Exposition - IMECE2007, Seattle, USA, 2007.

[18] C.C. Wang, C.K. Chen, Forced convection in a wavy-wall channel, Int. J. Heat Mass Transfer 45 (2002) 2587-2595.

[19] C.W. Tan, M.J. Normandia, Low Peclet number diffusion in narrow rectangular channels, Lett. Heat Mass Transfer 2 (1975) 259-266. 